\title{
1. Extended Data
}

Complete the Inventory below for all Extended Data figures.

\begin{tabular}{|c|c|c|c|}
\hline Figure \# & $\begin{array}{l}\text { Figure title } \\
\text { One sentence only }\end{array}$ & $\begin{array}{l}\text { Filename } \\
\text { This should be the } \\
\text { name the file is } \\
\text { saved as when it is } \\
\text { uploaded to our } \\
\text { system. Please } \\
\text { include the file } \\
\text { extension. i.e.: } \\
\text { Smith_ED_Fig1.jpg }\end{array}$ & $\begin{array}{l}\text { Figure Legend } \\
\text { If you are citing a reference for the first time in } \\
\text { these legends, please include all new references in } \\
\text { the Online Methods References section, and carry on } \\
\text { the numbering from the main References section of } \\
\text { the paper. }\end{array}$ \\
\hline $\begin{array}{l}\text { Extended Data } \\
\text { Fig. } 1\end{array}$ & $\begin{array}{l}\text { Correlation } \\
\text { between } \\
\text { transversal and } \\
\text { longitudinal } \\
\text { MSOT } \\
\text { collagen }{ }_{\text {mean/max }} \\
\text { signals }\end{array}$ & $\begin{array}{l}\text { Regensburger } \\
\text { ED_Fig1.jpg }\end{array}$ & $\begin{array}{l}\text { Each independent muscle was analyzed for } \\
\text { its transversal and longitudinal MSOT } \\
\text { collagen } n_{\text {mean }}(\mathbf{a}) \text { and collagen } \\
\text { max }(\mathbf{b}) \text { signal. } \\
\text { Correlations between longitudinal and } \\
\text { transversal MSOT collagen } n_{\text {mean } / \text { max }} \text { signals } \\
\text { are given by Spearman correlation } \\
\text { coefficient }\left(\mathrm{r}_{\mathrm{s}}\right) \text {. Two-tailed test. Linear } \\
\text { regression lines are in black. P values } \\
\leq 0.05 \text { were considered statistically } \\
\text { significant. } \mathrm{n}=316 \text { muscle regions ( } \mathrm{n}= \\
159 \text { transversal/n = } 157 \text { longitudinal } \\
\text { independent muscle regions) in } \mathrm{n}=20 \\
\text { biologically independent subjects }(\mathrm{n}=10 \\
\mathrm{HV} / \mathrm{n}=10 \text { DMD patients). }\end{array}$ \\
\hline $\begin{array}{l}\text { Extended Data } \\
\text { Fig. } 2\end{array}$ & $\begin{array}{l}\text { Standard B- } \\
\text { mode } \\
\text { ultrasound } \\
\text { imaging }\end{array}$ & $\begin{array}{l}\text { Regensburger_ } \\
\text { ED_Fig2.jpg }\end{array}$ & $\begin{array}{l}\text { Representative examples of transversal } \\
\text { and longitudinal B-mode ultrasound } \\
\text { imaging of quadriceps femoris muscles in } \\
\text { a HV and in a patient with DMD. A } \\
\text { representative result for a HV and a DMD } \\
\text { from } \mathrm{n}=160 \text { independent muscle regions } \\
(\mathrm{n}=80 \mathrm{HV} / \mathrm{n}=80 \mathrm{DMD} \text { ) of } \mathrm{n}=20 \\
\text { biologically independent subjects ( } \mathrm{n}=10 \\
\mathrm{HV} / \mathrm{n}=10 \mathrm{DMD} \text { patients) with similar } \\
\text { results is shown. Scale bars, } 1 \mathrm{~cm} \text {. }\end{array}$ \\
\hline $\begin{array}{l}\text { Extended Data } \\
\text { Fig. } 3\end{array}$ & $\begin{array}{l}\text { Quantification } \\
\text { of } 2 D \text { and } 3 D \\
\text { MSOT } \\
\text { collagen } \\
\text { signals in WT } \\
\text { and DMD } \\
\text { muscles over } \\
\text { time }\end{array}$ & $\begin{array}{l}\text { Regensburger_ } \\
\text { ED_Fig3.jpg }\end{array}$ & $\begin{array}{l}\text { Quantification of 2D (a) and 3D (b) MSOT } \\
\text { collagen }_{\max } \text { signals in WT and DMD piglet } \\
\text { muscles over time. WT and DMD MSOT } \text { collagen }_{\max } \text { signals of independent piglet } \\
\text { muscles of all animals were compared } \\
\text { with each other at weeks 1, 2, 3, and } 4 \text { of } \\
\text { age. Each filled circle represents one MSOT } \\
\text { signal per independent muscle region (n = }\end{array}$ \\
\hline
\end{tabular}




\begin{tabular}{|c|c|c|c|}
\hline & & & 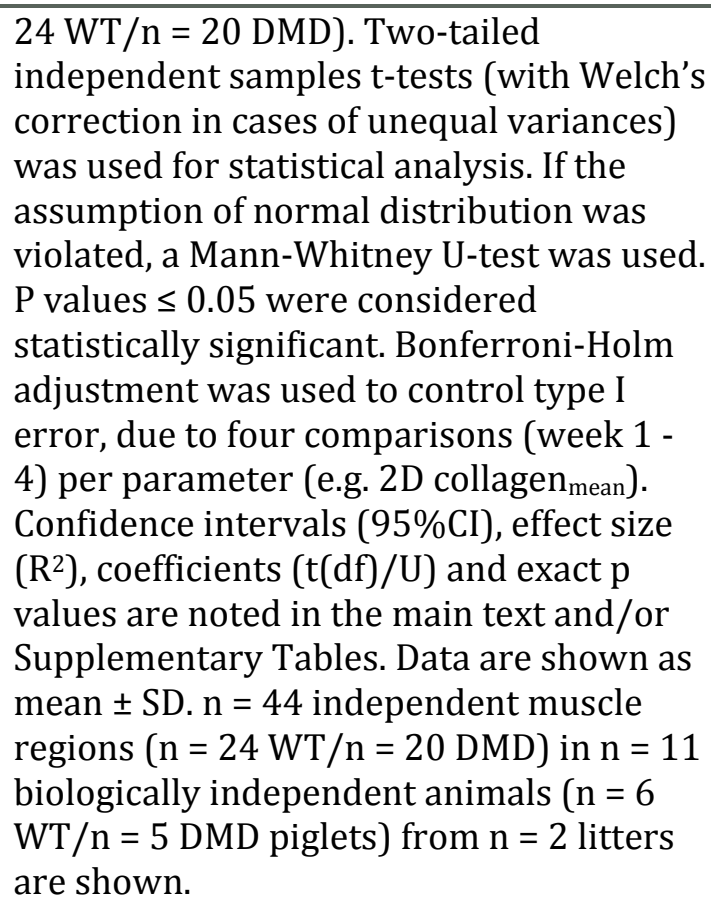 \\
\hline $\begin{array}{l}\text { Extended Data } \\
\text { Fig. } 4\end{array}$ & $\begin{array}{l}\text { Quantification } \\
\text { of 2D and 3D } \\
\text { MSOT } \\
\text { collagen }_{\text {max }} \\
\text { signals in WT } \\
\text { and DMD piglet } \\
\text { muscles over } \\
\text { time }\end{array}$ & $\begin{array}{l}\text { Regensburger_ } \\
\text { ED_Fig4.jpg }\end{array}$ & $\begin{array}{l}\text { 2D (a) and 3D (b) MSOT collagen } \text { max } \\
\text { signals of independent piglet muscles of } \\
\text { surviving animals were compared with } \\
\text { each other at weeks } 1,2,3 \text {, and } 4 \text { of age. } \\
\text { Each filled circle/square represents the } \\
\text { mean } \pm \text { SD MSOT signal of independent } \\
\text { muscle regions over the course of the } \\
\text { experiment ( } \mathrm{n}=12 \mathrm{WT} / \mathrm{n}=8 \mathrm{DMD}) .2 \mathrm{D} \\
\text { MSOT parameters were analyzed by post- } \\
\text { hoc Tukey's HSD following a mixed-effects } \\
\text { models due to missing values in week } 1 \\
\text { (litter } 1 \text { ). p values } \leq 0.05 \text { were considered } \\
\text { statistically significant. } 3 \mathrm{D} \text { MSOT collagen } \\
\text { parameters were analyzed by Tukey's } \\
\text { honestly significant difference tests } \\
\text { following a two-way (mixed design) } \\
\text { ANOVA; Data are shown as mean } \pm \text { SD. } \mathrm{n}= \\
20 \text { independent muscle regions ( } \mathrm{n}=12 \\
\text { WT } / \mathrm{n}=8 \text { DMD) in } \mathrm{n}=5 \text { biologically } \\
\text { independent animals ( } \mathrm{n}=3 \text { WT } / \mathrm{n}=2 \\
\text { DMD piglets) from } \mathrm{n}=2 \text { litters are shown. }\end{array}$ \\
\hline $\begin{array}{l}\text { Extended Data } \\
\text { Fig. } 5\end{array}$ & $\begin{array}{l}\text { Overview of } \\
\text { independent } \\
\text { muscle regions } \\
\text { in each DMD } \\
\text { piglet over time }\end{array}$ & $\begin{array}{l}\text { Regensburger_ } \\
\text { ED_Fig5.jpg }\end{array}$ & $\begin{array}{l}\text { 2D }(\mathbf{a}, \mathbf{b}) \text { and 3D }(\mathbf{c}, \mathbf{d}) \text { MSOT } \\
\text { collagen } \text { mean/max }_{\text {magnals of each }} \\
\text { independent muscle region of all surviving } \\
\text { DMD piglets over the course of the } \\
\text { experiment. Each icon represents one } \\
\text { independent muscle over the time period, }\end{array}$ \\
\hline
\end{tabular}




\begin{tabular}{|c|c|c|c|}
\hline & & & $\begin{array}{l}\text { connected by a colored line (weeks } 1,2,3 \text {, } \\
\text { and } 4 \text { of age). SR, shoulder right (yellow } \\
\text { line); LR, leg right (blue line); SL, shoulder } \\
\text { left (purple line); LL, leg left (green line). } n \\
=8 \text { independent muscle regions ( } n=4 \text { in } \\
\text { DMD-number- } 2 / n=4 \text { in DMD-number-5) } \\
\text { of } n=2 \text { biologically independent animals } \\
\text { ( } n=2 \text { DMD piglets) from } n=2 \text { litters are } \\
\text { shown. }\end{array}$ \\
\hline $\begin{array}{l}\text { Extended Data } \\
\text { Fig. } 6\end{array}$ & $\begin{array}{l}\text { Overview of the } \\
\text { mean MSOT } \\
\text { collagen } \\
\text { signals per } / \text { max } \\
\text { individual } \\
\text { piglet over time }\end{array}$ & $\begin{array}{l}\text { Regensburger } \\
\text { ED_Fig6.jpg }\end{array}$ & $\begin{array}{l}\text { Mean 2D (a, b) and mean 3D (c, d) MSOT } \\
\text { collagen } \text { mean } / \text { max signals per individual }_{\text {piglet over time (weeks } 1,2,3 \text {, and } 4 \text { of }} \\
\text { age). Each filled circle represents the mean } \\
\pm \text { SD MSOT signal of an independent piglet } \\
\text { over the course of the experiment ( } \mathrm{n}= \\
6 \mathrm{WT} / \mathrm{n}=5 \mathrm{DMD}) . \mathrm{n}=11 \text { biologically } \\
\text { independent animals }(\mathrm{n}=6 \mathrm{WT} / \mathrm{n}=5 \mathrm{DMD} \\
\text { piglets) from } \mathrm{n}=2 \text { litters are shown. }\end{array}$ \\
\hline $\begin{array}{l}\text { Extended Data } \\
\text { Fig. } 7\end{array}$ & $\begin{array}{l}\text { Standardizati } \\
\text { on and } \\
\text { positioning of } \\
\text { the detector } \\
\text { probe }\end{array}$ & $\begin{array}{l}\text { Regensburger_} \\
\text { ED_Fig7.jpg }\end{array}$ & $\begin{array}{l}\text { Examples of detector probe positioning } \\
\text { and MSOT scanning. Exact positioning of } \\
\text { the MSOT detector was standardized for } \\
\text { each anatomical region (Supplementary } \\
\text { Table 23) and marked with small labels } \\
\text { (e.g. red dots). Scanning of a 3-year-old } \\
\text { volunteer with the 2D MSOT detector is } \\
\text { presented. }\end{array}$ \\
\hline
\end{tabular}

Delete rows as needed to accommodate the number of figures (10 is the maximum allowed).

2. Supplementary Information:

\author{
A. Flat Files
}

12

\title{
13
}

Complete the Inventory below for all additional textual information and any additional Supplementary Figures, which should be supplied in one

\section{5 combined PDF file.}

\begin{tabular}{|l|l|l|l|}
\hline Item & Present? & $\begin{array}{l}\text { Filename } \\
\text { This should be the } \\
\text { name the file is saved } \\
\text { as when it is uploaded }\end{array}$ & $\begin{array}{l}\text { A brief, numerical description of file } \\
\text { contents. } \\
\text { i.e.: Supplementary Figures 1-4, Supplementary }\end{array}$ \\
\hline
\end{tabular}




\begin{tabular}{|l|l|l|l|}
\hline & & $\begin{array}{l}\text { to our system, and } \\
\text { should include the file } \\
\text { extension. The } \\
\text { extension must be } \\
\text {.pdf }\end{array}$ & Discussion, and Supplementary Tables 1-4. \\
\hline $\begin{array}{l}\text { Supplementary } \\
\text { Information }\end{array}$ & Yes & $\begin{array}{l}\text { 2_Supplementar } \\
\text { y } \\
\text { Appendix_V2.0. } \\
\text { pdf }\end{array}$ & Supplementary Tables 1-23 \\
\hline Reporting Summary & Yes & $\begin{array}{l}\text { reportingsumma } \\
\text { ry_1572018146 } \\
\text { 5.pdf }\end{array}$ & \\
\hline
\end{tabular}

21 Complete the Inventory below for all additional Supplementary Files

22 that cannot be submitted as part of the Combined PDF.

\begin{tabular}{|c|c|c|c|}
\hline Type & $\begin{array}{l}\text { Number } \\
\text { If there are multiple files of } \\
\text { the same type this should be } \\
\text { the numerical indicator. i.e. } \\
\text { "1" for Video 1, "2" for } \\
\text { Video 2, etc. }\end{array}$ & $\begin{array}{l}\text { Filename } \\
\text { This should be the name the } \\
\text { file is saved as when it is } \\
\text { uploaded to our system, and } \\
\text { should include the file } \\
\text { extension. i.e.: Smith_ } \\
\text { Supplementary_Video_1.mov }\end{array}$ & $\begin{array}{l}\text { Legend or Descriptive } \\
\text { Caption } \\
\text { Describe the contents of the } \\
\text { file }\end{array}$ \\
\hline Supplementary Video & & $\begin{array}{l}\text { NatMed_MSOT_DMD.m } \\
\text { ov }\end{array}$ & $\begin{array}{l}\text { Video - Real-time 2D } \\
\text { MSOT imaging in } \\
\text { newborn piglets } \\
\text { The video shows a } \\
\text { halved screen. On the } \\
\text { left side live imaging of } \\
\text { a piglet is presented. } \\
\text { The detector was placed } \\
\text { on the thigh (biceps } \\
\text { femoris muscle) of the } \\
\text { piglet. The right video } \\
\text { shows simultaneous } \\
\text { MSOT imaging. } \\
\text { Spectral unmixing for } \\
\text { collagen (turquoise) and } \\
\text { lipids (yellow) is } \\
\text { overlaid to RUCT } \\
\text { images. A }\end{array}$ \\
\hline
\end{tabular}


Add rows as needed to accommodate the number of files.

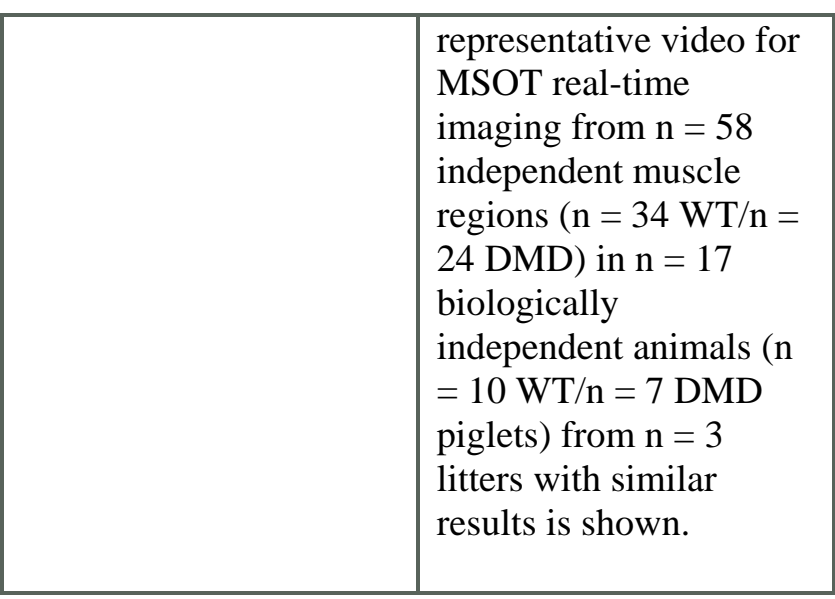

\section{Source Data}

Complete the Inventory below for all Source Data files.

\begin{tabular}{|c|c|c|}
\hline Figure & $\begin{array}{l}\text { Filename } \\
\text { This should be the name the file is } \\
\text { saved as when it is uploaded to our } \\
\text { system, and should include the file } \\
\text { extension. i.e.: } \\
\text { Smith_SourceData_Fig1.xls, or Smith_ } \\
\text { Unmodified_Gels_Fig1.pdf }\end{array}$ & $\begin{array}{l}\text { Data description } \\
\text { i.e.: Unprocessed Western Blots and/or gels, Statistical } \\
\text { Source Data, etc. }\end{array}$ \\
\hline Source Data Fig. 1 & Source_Data_Fig_1.xlsx & Statistical Source Data \\
\hline Source Data Fig. 2 & Source_Data_Fig_2.xlsx & Statistical Source Data \\
\hline Source Data Fig. 3 & Source_Data_Fig_3.xlsx & Statistical Source Data \\
\hline Source Data Fig. 4 & Source_Data_Fig_4.xlsx & Statistical Source Data \\
\hline Source Data Fig. 5 & Source_Data_Fig_5.xlsx & Statistical Source Data \\
\hline Source Data Fig. 6 & Source_Data_Fig_6.xlsx & Statistical Source Data \\
\hline \multicolumn{3}{|l|}{ Source Data Fig. 7} \\
\hline \multicolumn{3}{|l|}{ Source Data Fig. 8} \\
\hline $\begin{array}{l}\text { Source Data } \\
\text { Extended Data } \\
\text { Fig. } 1\end{array}$ & $\begin{array}{l}\text { Source_Data_Extended_Dat } \\
\text { a_Fig_1.xlsx }\end{array}$ & Statistical Source Data \\
\hline \multicolumn{3}{|l|}{$\begin{array}{l}\text { Source Data } \\
\text { Extended Data } \\
\text { Fig. } 2\end{array}$} \\
\hline Source Data & Source_Data_Extended_Dat & Statistical Source Data \\
\hline
\end{tabular}




\begin{tabular}{|l|l|l|}
\hline $\begin{array}{l}\text { Extended Data } \\
\text { Fig. } 3\end{array}$ & a_Fig_3.xlsx & \\
\hline $\begin{array}{l}\text { Source Data } \\
\text { Extended Data } \\
\text { Fig. } 4\end{array}$ & $\begin{array}{l}\text { Source_Data_Extended_Dat } \\
\text { a_Fig_4.xlsx }\end{array}$ & Statistical Source Data \\
\hline $\begin{array}{l}\text { Source Data } \\
\text { Extended Data } \\
\text { Fig. 5 }\end{array}$ & $\begin{array}{l}\text { Source_Data_Extended_Dat } \\
\text { a_Fig_5.xlsx }\end{array}$ & Statistical Source Data \\
\hline $\begin{array}{l}\text { Source Data } \\
\text { Extended Data } \\
\text { Fig. } 6\end{array}$ & Source_Data_Extended_Dat & Statistical Source Data \\
\hline
\end{tabular}

\section{for Duchenne muscular dystrophy}

37 Adrian P. Regensburger, M.D. ${ }^{1}$, Lina M. Fonteyne, D.V.M. ${ }^{2}$, Jörg Jüngert, M.D. ${ }^{1}$, Alexandra L. Wagner, M.D. ${ }^{1}$, Teresa Gerhalter, $\mathrm{PhD}^{3,4,5}$, Armin M Nagel, $\mathrm{PhD}^{3,4,5}$, Rafael Heiss, M.D. ${ }^{3}$, Florian Flenkenthaler, $\mathrm{PhD}^{2}$, Matthias Qurashi ${ }^{6}$, Markus F. Neurath, M.D. ${ }^{6,7}$, Nikolai Klymiuk, PhD², Elisabeth Kemter,

${ }^{1}$ Department of Pediatrics and Adolescent Medicine, University Hospital Erlangen, Friedrich-AlexanderUniversität Erlangen-Nürnberg (FAU), Erlangen, Germany

${ }^{2}$ Institute for Molecular Animal Breeding and Biotechnology, and Laboratory for Functional Genome Analysis (LAFUGA), Gene Center, Ludwig-Maximilian-University Munich, Munich, Germany

${ }^{3}$ Institute of Radiology, University Hospital Erlangen, Friedrich-Alexander-Universität ErlangenNürnberg (FAU), Erlangen, Germany

${ }^{4}$ Institute of Medical Physics, Friedrich-Alexander-Universität Erlangen-Nürnberg (FAU), Erlangen, Germany

${ }^{5}$ Division of Medical Physics in Radiology, German Cancer Research Center (DKFZ), Heidelberg, Germany

${ }^{6}$ Department of Medicine 1, University Hospital Erlangen, Friedrich-Alexander-Universität Erlangen-

55 Nürnberg (FAU), Erlangen, Germany 
$56 \quad{ }^{7}$ Ludwig Demling Center of Excellence, Friedrich-Alexander-Universität Erlangen-Nürnberg (FAU),

57 Erlangen, Germany

$58{ }^{8}$ Erlangen Graduate School in Advanced Optical Technologies (SAOT), Friedrich-Alexander-Universität

59 Erlangen-Nürnberg (FAU), Erlangen, Germany

60

61

$62 \dagger$ shared authorship

$63 *$ Corresponding author

64 Ferdinand Knieling, M.D.

65 Pediatric Experimental and Translational Imaging Laboratory (PETI-Lab)

66 Department of Pediatrics and Adolescent Medicine

67 University Hospital Erlangen

68 Friedrich-Alexander-University of Erlangen-Nürnberg

69 Loschgestraße 15

7091054 Erlangen, Germany

71

72 Phone: +49 $913185-33118$

73 Fax: +49 $913185-33113$

74 Mail: Ferdinand.Knieling@uk-erlangen.de

75 
77 Biomarkers for monitoring disease progression and response to therapy are lacking for muscle

78 diseases such as Duchenne muscular dystrophy. Non-invasive in vivo molecular imaging with

79 multispectral optoacoustic tomography (MSOT) utilizes pulsed laser light to induce acoustic

80 pressure waves, enabling the visualization of endogenous chromophores. Here, we describe a

81 novel application of MSOT, in which illumination in the near- and extended near-infrared range

82 (NIR and exNIR) from 680-1100 nm enables the visualization and quantification of collagen

83 content. We first demonstrated the feasibility of this approach to non-invasively quantify tissue

84 fibrosis in longitudinal studies in a large-animal DMD model in pigs, and then applied this

85 approach to pediatric patients (NCT03490214). MSOT-derived collagen content measurements

86 in skeletal muscle were highly correlated to the functional status of the patients and provided

87 additional information on molecular features as compared to magnetic resonance imaging. This

88 study highlights the potential of MSOT imaging as a non-invasive, age-independent biomarker

89 for the implementation and monitoring of newly-developed therapies in muscular diseases. 


\section{$91 \quad$ Introduction}

92 Duchenne muscular dystrophy (DMD) is the most common lethal inherited X-chromosomal

93 muscular disease occurring in one of 3,800-6,000 live male births ${ }^{1}$. Initially, affected boys

94 develop normally, but at the age of four to five years loss of functional muscle mass becomes

95 apparent. Within a few years, relevant muscle and tendon shortening lead to muscular weakness

96 resulting in loss of ambulation around the age of ten, finally ending in respiratory and cardiac

97 failure in the third decade. DMD is caused by loss-of-function mutations in the dystrophin gene

98 leading to dystrophin deficiency and resulting in muscular degeneration, followed by

99 inflammation and fatty and fibrotic transformation ${ }^{2-4}$. Promising new therapeutic approaches

100 aiming to restore disturbed molecular mechanisms by enabling the ribosomal read-through of

101 premature stop codons ${ }^{5,6}$ or exon skipping ${ }^{7}$ have been approved, and others such as viral gene

102 therapy, utrophin modulators ${ }^{8}$ and CRISPR/Cas-mediated correction of $D M D$ mutations provide

103 promising early results ${ }^{9}$. Until now, primary outcome measures are based on manual ${ }^{10}$ and

104 quantitative muscle examinations ${ }^{11}$ as well as timed function tests ${ }^{5-7,12}$. Furthermore, magnetic

105 resonance imaging (MRI) ${ }^{13-15}$ and magnetic resonance spectroscopy (MRS) ${ }^{16-18}$ have shown

106 potential as non-invasive imaging techniques for quantification of disease pathology and

107 progression in DMD. However, these and other techniques have limited applicability due to

108 considerable acquisition times ${ }^{14,18}$, a required sedation in early childhood and uncooperative

109 patients, as well as the effort towards nationwide availability of novel standardized imaging

110 protocols ${ }^{19}$. Until now, a standardized 6-minute walk test (6-MWT) is one of the main primary

111 outcome measures to assess the effects of pharmaceutical interventions ${ }^{5-7,20,21}$. However,

112 physical examinations rely on active cooperation and the individual performance on the day,

113 which might limit their diagnostic validity. To overcome an unmet clinical need for independent 
114 easy-to-apply prognostic biomarkers ${ }^{22}$ and to enable guidance for earliest therapeutic

115 interventions during infancy, novel non-invasive diagnostic approaches are required.

116 Multispectral Optoacoustic Tomography (MSOT) is an emerging imaging modality capable of

117 non-invasively visualizing the distribution of endogenous absorbers by initiating laser-induced

118 thermoelastic expansion and detection of resulting pressure waves ${ }^{23,24}$. Further, the clinical

119 usability for the detection of endogenous molecular chromophores, such as (de-)oxygenated

120 hemoglobin, oxygen saturation, and melanin has already been demonstrated in melanoma lymph

121 nodes ${ }^{25}$, breast cancer ${ }^{26,27}$, melanoma ${ }^{28}$ and chronic intestinal inflammation ${ }^{29,30}$.

122 The utilization of wavelengths in the extended near-infrared range (exNIR) would also enable

123 the visualization of lipids and collagens ${ }^{31-36}$. This report provides the first insight into how

124 MSOT using exNIR illumination is capable of visualizing fibrotic muscular transformation in 125 vivo suggesting its application as a novel non-invasive imaging biomarker in muscular 126 dystrophy.

\section{Results}

\section{NIR/exNIR MSOT imaging detects and separates collagen signals}

130 MSOT uses spectral unmixing to identify different endogenous chromophores (Fig. 1a). To

131 prove the unmixing algorithm and signal separation for collagens, a custom-made phantom was

132 built. First, solutions of purified human collagens (I, III, and IV) were imaged every $5 \mathrm{~nm}$ from

$133 \quad 660-1200 \mathrm{~nm}$. The separated optoacoustic spectra of each collagen peaked similarly between 980

134 and $1000 \mathrm{~nm}$, clearly distinguishable from hemoglobin, lipid, and water signals (Fig. 1b). For

135 further validation, normalized spectra of ex vivo porcine tendons (mainly collagen I), collagen I

136 solutions, and the spectra derived from literature ${ }^{31}$ were compared. Therefore, a region of 
137 interest (ROI) was outlined in the reflective ultrasound computed tomography (RUCT), which is

138 used to anatomically guide the investigator during imaging (Fig. 1c). When using a translatable 139 setup limited to a maximum of 11 wavelengths $(680,700,730,760,800,850,920,1000,1030$,

140 1064, and 1100 nm, Fig. 1c) the collagen spectra of the tendon and the purified collagen I

141 resembled those reported in the literature (collagen peak at $\sim 1000 \mathrm{~nm}$ ) (Fig. 1c) ${ }^{31}$. Furthermore,

142 in vivo imaging of tendons was carried out, confirming our spectral unmixing approach, by

143 showing specific unmixed collagen signals mainly within the tendon (Fig. 1d).

144

145 Collagen detection in a porcine DMD model

146 Subsequently, 2D MSOT imaging was performed in a translational porcine model of DMD (Fig.

147 2a and Supplementary Video). In total $n=58$ scans from independent muscle regions $(n=34$

148 in wild type piglets (WT)/n = 24 in DMD piglets) were acquired from $n=17$ piglets $(\mathrm{n}=10$

$149 \mathrm{WT} / \mathrm{n}=7 \mathrm{DMD})$. Using ultrasound guidance, a region of interest (ROI) was drawn within the

150 examined muscle to quantify the MSOT parameters, such as the mean and the maximum

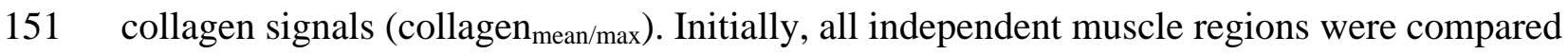

152 between groups ( $\mathrm{n}=34 \mathrm{WT} / \mathrm{n}=24 \mathrm{DMD}$ muscles). A significant difference for the

153 collagen $_{\text {mean/max }}$ signal was observed (collagen mean $_{1} 14.41 \pm 2.66$ a.u. vs. $23.14 \pm 3.87$ a.u., $\mathrm{p}=$

$1541.18 \times 10^{-11}, \mathrm{t}(\mathrm{df})=9.57$ (38), 95\%CI $6.88-10.57, \mathrm{R}^{2}=0.71$; collagen max $_{2} 27.68 \pm 2.72$ a.u. vs.

$15541.05 \pm 7.43$ a.u., $\left.\mathrm{p}=4.38 \times 10^{-9},(\mathrm{df})=8.43(27), 95 \% \mathrm{CI} 10.12-16.63, \mathrm{R}^{2}=0.72\right)$. Second,

156 when comparing a mean collagen signal per independent piglet between groups $(\mathrm{n}=10 \mathrm{WT} / \mathrm{n}=7$

157 DMD piglets), the collagen mean/max $_{\text {signals were similarly increased (collagen }}$ mean: $14.23 \pm 1.96$

158 a.u. vs. $22.67 \pm 3.59$ a.u., $p=1.51 \times 10^{-5}$, (df) = 6.27 (15), 95\%CI $5.57-11.31, \mathrm{R}^{2}=0.72$;

159 collagen $_{\max }: 27.70 \pm 1.67$ a.u. vs. $41.01 \pm 5.16$ a.u., $p=1.39 \times 10^{-6}$, (df) $=7.69(15), 95 \% \mathrm{CI}$ 
$\left.59.62-17.00, R^{2}=0.80\right)$. There was no difference in signal levels of deoxygenated $\left(H b_{R}\right)$,

161 oxygenated $\left(\mathrm{HbO}_{2}\right)$ and total hemoglobin $\left(\mathrm{Hb}_{\text {total }}\right)(\mathrm{p}>0.05$, Fig. $2 \mathbf{b}$ and Supplementary Table

1621 and 2).

163 An exploratory receiver operator characteristic (ROC) analysis revealed excellent ability of

164 MSOT-derived collagen signals to distinguish healthy from diseased muscles ( $\mathrm{n}=34 \mathrm{WT} / \mathrm{n}=24$

165 DMD muscle regions) (collagen ${ }_{\text {mean }}$ : AUC 0.98, 95\%CI $0.95-1.00, \mathrm{p}=6.06 \times 10^{-10}$; collagen $_{\max }$ :

166 AUC 0.98, 95\%CI $0.96-1.00, p=4.96 \times 10^{-10}$, Fig. 2c). Corresponding tissue specimens were

167 taken for validation in which ex vivo histopathology revealed muscular dystrophy and a

168 qualitative increase in collagen formation in diseased animals (Fig. 2d). In total, $n=18$ WT

169 muscles of $\mathrm{n}=6$ piglets and $\mathrm{n}=12$ DMD muscles of $\mathrm{n}=3$ piglets were analyzed quantitatively

170 by the positive stained collagen area on Trichrome (TriC) and Sirius Red (SirR) stained

171 histological sections or the ratio between total collagen and total protein (TC/TP). One WT

172 muscle could not be histologically quantified. All corresponding analyses showed significant

173 differences between the cohorts suggesting elevated values in DMD piglets (means: TriC: 5.35\%

174 vs. $12.63 \%, \mathrm{p}=4.08 \times 10^{-5},(\mathrm{df})=5.59(16), 95 \%$ CI $4.52-10.05, \mathrm{R}^{2}=0.66$; SirR: $8.44 \%$ vs.

$17516.23 \%, \mathrm{p}=0.0003,(\mathrm{df})=4.56(16), 95 \% \mathrm{CI} 4.17-11.39, \mathrm{R}^{2}=0.56$; and TC/TP: $46.83 \mu \mathrm{g}$

176 collagen/mg protein vs. $64.95 \mu$ g collagen/mg protein, $\mathrm{p}=0.0240,(\mathrm{df})=2.39(28), 95 \% \mathrm{CI} 2.56-$

$17733.68, R^{2}=0.19$, respectively, Fig. 2e-g). Additionally, highly significant correlations to MSOT

178 collagen signals were found (TriC: $r_{s}=0.58, p=0.0010,95 \%$ CI $0.26-0.78$, SirR: $r_{s}=0.65, p=$

$1790.0001,95 \%$ CI $0.37-0.83$ and TC/TP: $\mathrm{r}_{\mathrm{s}}=0.53, \mathrm{p}=0.0027,95 \%$ CI $0.20-0.75$, respectively,

180 Fig. 2e-g).

181

182 Two-dimensional collagen detection in DMD patients and healthy volunteers 
183 To study our approach in patients, we enrolled $n=10$ DMD patients and $n=10$ age- and gender-

184 matched healthy volunteers (HV) in a first-in pediatric trial. Mean age of affected boys was $7.1 \pm$

1851.6 years and $7.3 \pm 2.2$ years in matched HV (detailed clinical data is shown in the

186 Supplementary Table 3).

187 In total $\mathrm{N}=320$ scans (transversal and longitudinal scans of eight muscles per participant) were

188 obtained. $\mathrm{N}=4$ scans were excluded for the following reasons: $\mathrm{N}=1$ broken scan, $\mathrm{N}=3$ region

189 of interest (ROI) was beyond the depth limit of the detector (below $3.5 \mathrm{~cm}$ ). The average scan

190 time for 2D and 3D images was $6.3 \pm 0.8 \mathrm{~min}$ in DMD patients and $6.7 \pm 0.6 \mathrm{~min}$ in $\mathrm{HV}$.

191 Exemplary in vivo imaging (Fig. 3a) and transversal images of a single HV and DMD patient are

192 presented in Fig. 3b, illustrating a qualitative difference of collagen signal intensity in every

193 muscle region between both groups. In accordance with our preclinical model, each independent

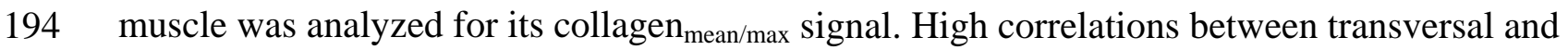

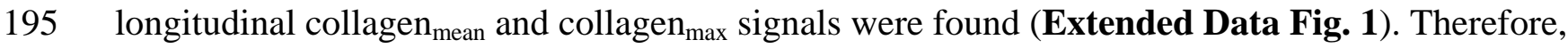

196 only transversal images were used for further analyses. All independent muscle regions $(\mathrm{n}=80$

$197 \mathrm{HV} / \mathrm{n}=79$ DMD muscles), showed statistically significant differences between both groups for

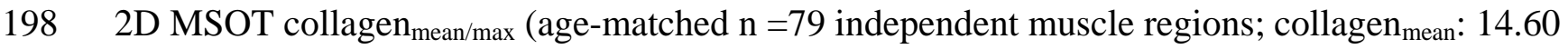

$199 \pm 4.42$ a.u. vs. $24.72 \pm 5.92$ a.u., $\mathrm{p}<1.0 \times 10^{-15}$, 95\%CI $8.50-11.76$, t(df) $=12.37(78), \mathrm{R}^{2}=0.66$

200 and collagen $\max _{\text {ax }} 26.55 \pm 6.16$ a.u. vs. $40.52 \pm 7.71$ a.u., $\mathrm{p}<1.0 \times 10^{-15}, 95 \% \mathrm{CI} 10.40-16.28, \mathrm{~W}^{-}$ $201=-17)$.

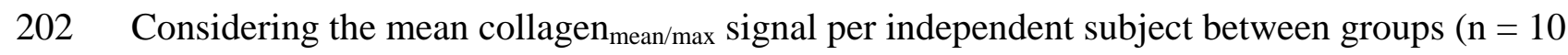

$203 \mathrm{HV} / \mathrm{n}=10 \mathrm{DMD}$ patients), significant differences in matched comparison of the mean

204 (collagen $_{\text {mean }}: 14.59 \pm 2.52$ a.u. vs. $24.70 \pm 2.77$ a.u., $p=1.78 \times 10^{-5}, 95 \%$ CI $7.33-12.90, t(d f)=$

205 8.22(9), $\mathrm{R}^{2}=0.88$ ) and the maximum collagen content (collagen $\max _{2} 26.59 \pm 3.51$ a.u. vs. $40.48 \pm$ 
3.81 a.u., $p=5.17 \times 10^{-6}, 95 \%$ CI $\left.10.61-17.18, t(d f)=9,57(9), R^{2}=0.91\right)$ were found (Fig. 3c).

207 There was no significant difference found in lipid, $\mathrm{Hb}_{\mathrm{R}}, \mathrm{HbO}_{2}$ and $\mathrm{Hb}_{\text {total }}$ signals between both

208 groups (Supplementary Table 4). ROC analysis of $n=80$ HV/n $=79$ DMD independent muscle

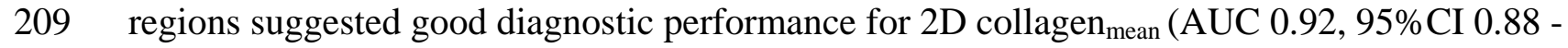

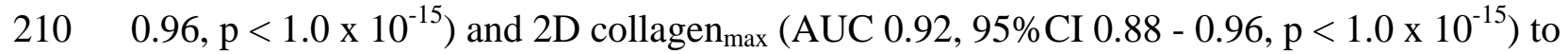

211 distinguish healthy from diseased muscles (Fig. 3d).

212

213 Three-dimensional MSOT imaging in DMD patients and healthy volunteers

214 Furthermore, we aimed to prove a volumetric (3D) MSOT imaging approach. A total of $\mathrm{N}=160$

215 3D scans from independent muscle regions ( $\mathrm{n}=80 \mathrm{HV} / \mathrm{n}=80$ DMD muscles) were acquired.

216 The 3D detector is designed as a cup (Fig. 4a) and operates at higher frequencies (8 MHz)

217 compared to the 2D detector (4 MHz). The heavy water couplet and a thinner detector foil,

218 enable a high resolution and a clear visualization of subcutaneous chromophores (Fig. $4 \mathbf{4 b}$ ).

219 Similar to our 2D analysis approach, 3D data sets demonstrated a significant difference of the

220 collagen $_{\operatorname{mean} / \max }$ signal in all anatomical independent muscle regions ( age-matched $\mathrm{n}=80$

221 independent muscle regions, $\mathrm{n}=80 \mathrm{HV} / \mathrm{n}=80$ DMD: collagen $_{\text {mean }} 5.39 \pm 2.20$ a.u. vs $11.41 \pm$

222 2.10 a.u., $\mathrm{p}<1.0 \times 10^{-15}, 95 \% \mathrm{CI} 5.28-6.76, \mathrm{t}(\mathrm{df})=16.09(79), \mathrm{R}^{2}=0.77$ and collagen $\max _{\max } 12.94 \pm$

2232.95 a.u. vs $23.66 \pm 6.46$ a.u., $\left.\mathrm{p}<1.0 \times 10^{-15}, 95 \% \mathrm{CI} 7.60-10.90, \mathrm{~W}^{-}=-2\right)$. The mean

224 collagen $_{\operatorname{mean} / \max }$ signal for each independent subject were analyzed and showed significant

225 differences (age-matched $\mathrm{n}=10 \mathrm{HV}$ and $\mathrm{n}=10 \mathrm{DMD}$ patients: collagen $\mathrm{mean} 5.39 \pm 0.80$ a.u. vs

$22611.41 \pm 1.01$ a.u., $\mathrm{p}=9.99 \times 10^{-8}, 95 \%$ CI $5.13-6.92$, $\mathrm{t}(\mathrm{df})=15.2(9), \mathrm{R}^{2}=0.96$ and collagen $_{\max }$

$22712.94 \pm 1.19$ a.u. vs $23.66 \pm 3.28$ a.u., $\mathrm{p}=2,01 \times 10^{-5}, 95 \% \mathrm{CI} 7.73-13.72$, $\mathrm{t}(\mathrm{df})=8.1(9), \mathrm{R}^{2}=$

228 0.88). In contrast to the scans using the 2D detector, there were significant differences in the 
optoacoustic signals for $\mathrm{Hb}_{\mathrm{R}}, \mathrm{HbO}_{2}$ and $\mathrm{Hb}_{\text {total }}$ using 3D-detection. In DMD subjects, $\mathrm{Hb}_{\mathrm{R}}, \mathrm{HbO}_{2}$

230 and $\mathrm{Hb}_{\text {total }}$ were significantly decreased (Fig. 4c and Supplementary Table 5). The mean

231 collagen content was inversely correlated with $\mathrm{Hb}_{\mathrm{R}}(\mathrm{n}=20$ independent patients $(\mathrm{n}=10 \mathrm{HV} / \mathrm{n}=$

232 10DMD; mean signal): $\left.\mathrm{r}_{\mathrm{s}}=-0.60, \mathrm{p}=0.0051,95 \% \mathrm{CI}-0.83-(-0.20)\right), \mathrm{HbO}_{2}(\mathrm{n}=20$ independent

233 patients $(\mathrm{n}=10 \mathrm{HV} / \mathrm{n}=10 \mathrm{DMD}$, mean signal $): \mathrm{r}_{\mathrm{s}}=-0.81, \mathrm{p}=1.67 \times 10^{-5}, 95 \% \mathrm{CI}-0.92-(-$

234 0.56) $)$ and $\mathrm{Hb}_{\text {total }}\left(\mathrm{n}=20\right.$ independent patients $\left(\mathrm{n}=10 \mathrm{HV} / \mathrm{n}=10 \mathrm{DMD}\right.$, mean signal): $\mathrm{r}_{\mathrm{s}}=-0.81$,

$\left.235 \mathrm{p}=1.78 \times 10^{-5}, 95 \% \mathrm{CI}-0.92-(-0.56)\right)$ contents of the muscle. ROC analysis demonstrated

236 excellent diagnostic performance of the 3D approach to distinguish healthy from diseased

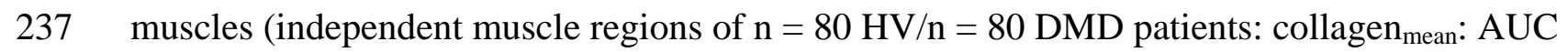

$238 \quad 0.98,95 \%$ CI $0.97-1.00, \mathrm{p}<1.0 \times 10^{-15}$ and collagen ${ }_{\max }$ : AUC 0.98, 95\%CI $0.96-1.00, \mathrm{p}<1.0 \mathrm{x}$

$\left.23910^{-15}\right)$ (Fig. 4d).

240

\section{MSOT has significant correlation to clinical standard assessments}

242 Every participant $(\mathrm{N}=20)$ underwent a standardized physical examination. Except the time for a

243 single rise from chair and muscle strength of the upper/lower distal extremities, all timed

244 function tests and manual muscle testing, showed significant between-group differences

245 (Supplementary Table 3). Notably, not all patients were able to complete all tests, due to

246 cognitive impairment, fatigue, or state of distraction.

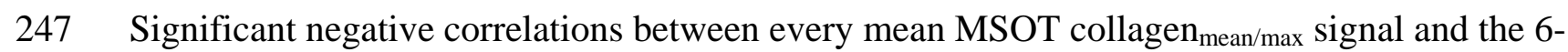

248 MWT were found $\left(n=20\right.$ independent subjects $(n=10 \mathrm{HV} / \mathrm{n}=10 \mathrm{DMD})$ : collagen $2 \mathrm{D}-\operatorname{mean} \mathrm{r}_{\mathrm{s}}=$ -

$2490.74, \mathrm{p}=0.0002,95 \% \mathrm{CI}-0.89-(-0.42)$; collagen $_{2 \mathrm{D}-\max } \mathrm{r}_{\mathrm{s}}=-0.69, \mathrm{p}=0.0007,95 \% \mathrm{CI}-0.87-(-$

250 0.35); collagen $3 D$-mean $r_{s}=-0.64, p=0.0023,95 \% C I-0.85-(-0.26)$ collagen $_{3 D-m a x} r_{s}=-0.71, p=$

$2510.0005,95 \%$ CI $-0.88-(-0.38))$. The other timed function tests showed similar consistent 
252 correlation with collagen signals, except single rise from chair and MRC of the lower distal

253 extremity. No correlation with age was observed (e.g. collagen ${ }_{2 D-m e a n} r_{s}=-0.06, p=0.79,95 \% C I$

$254-0.50-0.40) ;$, Fig. 5a and Supplementary Table 6). In addition, comparison of MSOT

255 collagen signals between the thigh and lower leg in DMD patients showed no overall significant

256 differences (Supplementary Table 7). In comparison to MSOT, a total of $n=320$ standard

257 ultrasound B-mode images ( $\mathrm{n}=160$ transversal $/ \mathrm{n}=160$ longitudinal images) were evaluated

258 (Extended Data Figure 2). Only transversal scans were used for statistical analyses.

259 Independent muscles of HV ( $\mathrm{n}=80)$ and DMD patients $(\mathrm{n}=80)$ showed different echogenicity,

260 texture, and scores on Heckmatt scale (Supplementary Table 8). A negative correlation

261 between the mean MSOT 2D collagen $n_{\text {mean }}$ and muscle echogenicity (Spearman $r_{s}=-0.53,6.08 x$

$26210^{-13}, 95 \%$ CI $-0.63-(-0.41)$ ), and a significant positive correlation between MSOT collagen and

263 Heckmatt scale (Spearman $r_{s}=0.38,9.39 \times 10^{-7}, 95 \%$ CI $0.23-0.51$, Supplementary Table 9)

264 were found. During all MSOT investigations, ultrasound, and physical examinations, no serious

265 adverse events were reported (Supplementary Table 10).

266 For further comparison, $n=5$ DMD patients underwent MRI of the right lower leg (Fig. 5b).

267 Total scan time was between 60 and 75 minutes. The time interval between MRI and MSOT

268 imaging was $2.4 \pm 1.34$ months (for details see Supplementary Table 11). No significant

269 correlation was found between MSOT collagen $n_{\text {mean }}(\mathrm{n}=5$ DMD patients) and fat fraction (FF),

270 water $T_{2}$, total tissue sodium concentration (TSC), and intracellular-weighted sodium signal

271 (ICwS), respectively. However, TSC and ICwS content measured by MRI and collagen signals

272 derived by MSOT showed a correlation but were not significant $\left(r_{s}=0.70, p=0.23,95 \%\right.$ CI $\left.n / a\right)$,

273 respectively) (Supplementary Table 12). Noteworthy, the patient with the highest FF (FF of 
274 0.374) showed the highest 2D MSOT lipid (920 nm) signal (Fig. 5b and Supplementary Table 275 11).

276

277 MSOT quantitatively visualizes early-stage disease progression

278 To demonstrate the feasibility of our approach for the in vivo monitoring of disease progression 279 in DMD, a longitudinal study in the DMD piglet model was conducted (Fig. 6a). From initial $\mathrm{n}=$ 28011 male piglets, $\mathrm{n}=5$ completed the full experimental protocol and were sacrificed after 4 times 281 consecutive weekly MSOT imaging. $\mathrm{n}=3$ DMD piglets died early for different reasons; thus $\mathrm{n}=$ 2823 corresponding WT piglets were sacrificedll other animals were sacrificed after imaging 283 procedures in week 4. (Fig. $6 \mathbf{b}$ ). $n=44$ independent muscles (biceps and triceps muscle of both 284 sides, Fig 6c) $(\mathrm{n}=24 \mathrm{WT} / \mathrm{n}=20 \mathrm{DMD})$ were investigated in week 1 , and $\mathrm{n}=20$ independent 285 muscles ( $\mathrm{n}=12 \mathrm{WT} / \mathrm{n}=8 \mathrm{DMD}$ ) were imaged through the whole experiment. In vivo MSOT 286 imaging revealed visible tissue changes in the DMD piglets over time by means of increased

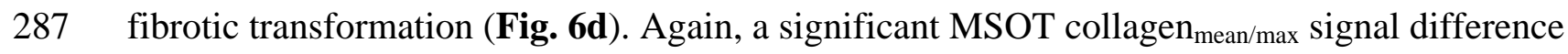
288 (2D and 3D) was found in all independent muscle regions of WT ( $n=24$ in week $1 / n=12$ in 289 week 2, 3, and 4) and DMD ( $\mathrm{n}=20$ in week $1 / \mathrm{n}=8$ in week 2, 3, and 4) piglets compared 290 between every week of the experiment (Fig. 6e, Extended Data Fig. 3, Supplementary Table

29113 and Supplementary Table 14). Comparison of the independent muscle regions ( $\mathrm{n}=12$

$292 \mathrm{WT} / \mathrm{n}=8 \mathrm{DMD})$ of the preserving $\mathrm{n}=5$ piglets $(\mathrm{n}=3 \mathrm{WT} / \mathrm{n}=2 \mathrm{DMD})$ showed a steady 293 increase of 2D and 3D MSOT collagen signals only in DMD over the time course of four weeks 294 (Fig. 6e, Extended Data Fig. 4-6, Supplementary Tables 15-18). At week 4, the end of the 295 study, the differences between both cohorts were clearly evident in vivo, ex vivo 296 macroscopically, and histologically (Fig. 6f and 6g). In the DMD cohort, histological and 
297 bioanalytical collagen quantification demonstrated an increased collagen deposition within four

298 weeks up to $248 \%$ (TriC \%, week 1 to week 4: $10.40 \pm 5.14$ to $25.80 \pm 7.93$, p $=4.94 \times 10^{-5}$, t(df)

$299=5.29(18), 95 \%$ CI 9.29-21.51, $\left.\mathrm{R}^{2}=0.61\right)$ and $170 \%($ SirR $\%$, week 1 to week $4: 19.59 \pm 5.40$ to

$\left.30033.30 \pm 5.71, \mathrm{p}=3.63 \times 10^{-5}, \mathrm{t}(\mathrm{df})=5.43(18), 95 \% \mathrm{CI} 8.41-19.01, \mathrm{R}^{2}=0.62\right)$ from baseline,

301 respectively. Collagen per protein content quantification increased up to 139\% (TC/TP $\mu \mathrm{g} / \mathrm{mg}$

302 week 1 to week 4: $77.57 \pm 38.80$ to $107.80 \pm 42.02$, $p=0.12$, t(df) $=1.65$ (18), 95\%CI -8.24 -

303 68.63, $\left.\mathbf{R}^{2}=0.13\right)$ (Fig. 6h).

304 To further confirm muscular fibrosis and the potential origin of MSOT signals, quantitative

305 proteome analysis was performed on $n=16$ snap-frozen independent tissue samples $(n=8 \mathrm{WT} / \mathrm{n}$

$306=8$ DMD) of $n=8$ independent piglets $(n=4 \mathrm{WT} / \mathrm{n}=4 \mathrm{DMD})$, showing clear separation of

307 proteomes according to age and genotype (Fig. 6i-k). In total, 2820 proteins were identified (Fig.

308 6k). Comparing collagens between WT and DMD piglets, collagen VI (COL6A1, 1.7-fold;

309 COL6A2 1.6-fold; COL6A3, 1.7-fold) was already enriched in DMD piglets in week one of life.

310 In the fourth week of life, besides the most abundant collagen VI, collagen III and collagen XIV

311 were also increased (COL3A1, 6.3-fold; COL14A1, 1.6-fold) (Supplementary Table 19 and

\section{Supplementary Table 20).}

\section{Discussion}

315 This translational approach suggests a potential role for MSOT as a novel in vivo contrast-agent

316 free and non-invasive imaging modality for the quantitative detection of collagen as a biomarker

317 in DMD.

318 In our previous work, we already demonstrated the capability of MSOT in near infrared range

319 (NIR) for disease monitoring in Crohn's disease by detecting different signal levels of 
320 hemoglobin as markers of intestinal inflammatory activity ${ }^{29,30}$. To date, the utilization of

321 wavelengths in the extended near-infrared range (exNIR) has so far only been reported in

322 experimental settings ${ }^{28,32,35,36}$ but, to the best of our knowledge, has never been applied in a

323 clinical trial - especially not in pediatrics.

324 Our findings suggest that quantitative assessment of collagen content in muscles with MSOT is a

325 suitable method for monitoring of degeneration involving fibrotic processes in vivo, as shown in

326 this study in a large animal model and a pediatric cohort. The extracted collagen spectra were in

327 agreement with previously reported optoacoustic spectra ${ }^{31,33-35}$; visualization and quantification

328 of collagen was feasible in all anatomical regions and showed significant differences between

329 diseased and healthy animals as well as in the human cohorts. Accelerated disease progression in

330 DMD piglets leading to early structural changes ${ }^{37}$ might explain comparable results of animal

331 and human findings in this study.

332 Currently, several new treatments for DMD are under investigation, but until now, there is an

333 unmet need for established objective monitoring techniques and age-independent biomarkers in

334 clinical practice ${ }^{13,22}$. So far, the 6-MWT is the most commonly used primary endpoint for

335 disease assessment in DMD but essentially requires active patient compliance ${ }^{5-7,20,21}$. The

336 complexity to accurately execute these tests and the dependence on cooperative behavior limit

337 the significance of muscular function tests to more adolescent patients. Most recent trials were

338 restricted to DMD patients aged over 5-7 years ${ }^{5-7}$, which prohibits conclusions on early

339 therapeutic interventions. In comparison to established imaging modalities like ultrasound

340 imaging ${ }^{38,39}$ and MRI ${ }^{40-43}$, MSOT is a target-specific quantitative, non-invasive, bedside

341 imaging modality. MRI is still limited by long image-acquisition times and requires

342 immobilization and, in early childhood, sedation. In our study, we demonstrated that MSOT can 
343 be performed in subjects down to 3 years of age, while minimal scan times ( $<7 \mathrm{~min}$ for 8

344 anatomical regions) suggest that it could even be performed from birth.

345 MRI protocols are further limited for detecting fibrosis specifically, since signal alterations in

346 T2-weighted, water-sensitive MRI images are nonspecific, due to the sensitivity to inflammation,

347 edema, fibrosis, as well as necrosis and the influence of corticosteroid treatments ${ }^{43}$. MRI has

348 shown potential for treatment monitoring ${ }^{42,43}$, and emerging protocols like ${ }^{23} \mathrm{Na}$ MRI might even

349 add further value ${ }^{18,42,44}$. The latter enables detection of increased sodium content, which

350 potentially also reflects elevated glycosaminoglycans (GAGs) ${ }^{45}$ and extracellular matrix (ECM)

351 production ${ }^{46,47}$. The similarities between increased MRI TSC and MSOT collagen content in our

352 study points towards an overlapping depiction of enriched ECM in DMD patients ${ }^{48}$. However,

353 the conclusions drawn in our study regarding MRI TSC are limited by the small sample size and

354 the heterogeneity of the DMD collective.

355 The slightly divergent imaging outcomes for the 2D and 3D detector might reflect different

356 technical designs of the detectors used in our study. The differences of deoxygenated and

357 oxygenated hemoglobin content of the muscle between HV and DMD patients and the negative

358 correlation of hemoglobin and collagens is in line with the pathophysiological mechanism of

359 muscular degeneration underlying DMD ${ }^{4}$. Notably, fatty transformation could not be

360 unequivocally detected using MSOT, most likely due to the high absorption of the subcutaneous

361 fat tissue compared to the relatively low fat fraction $(<10 \%)$ within the muscles of four of the

362 five investigated participants. However, this also reflects the findings in previous reports where

363 combined peri- and endomysial fibrosis were exceeding $30 \%{ }^{49}$, while intramuscular fat content

364 in similar aged DMD biceps femoris and quadriceps femoris muscle ranged only from $0.89 \pm$ 
$3650.70 \%{ }^{50}$ to $3.4 \pm 4.1 \%{ }^{49}$, respectively. In this regard, fatty infiltration is known to be distributed 366 very heterogeneously within single muscles of DMD patients ${ }^{51,52}$.

367 Dating the initiation of skeletal muscle degeneration back to intrauterine life ${ }^{53,54}$, Peverelli et al.

368 observed increased connective tissue proportions of 16.5\% in one-year-old DMD patients

369 compared to $3 \%$ in healthy subjects, rapidly peaking to $30 \%$ and more in the following years

$37049,50,55,56$. These findings support our concept to consider fibrosis as an early imaging target in

371 DMD.

372 As this was the first-in pediatric use of multispectral optoacoustic imaging, this study was

373 limited in scope and size. Nevertheless, 320 2D scans and 160 3D scans were recorded in this

374 exploratory study, which underlines the ability of MSOT as a highly specific and sensitive tool

375 for potential disease monitoring in DMD. Excellent imaging precision ${ }^{57}$, as well as

376 reproducibility and repeatability ${ }^{58}$ have been shown previously. We have demonstrated an age-

377 independent, significant negative correlation between MSOT collagen parameters and the

378 clinical muscle function. Considering results from our longitudinal experiments, MSOT might be

379 suitable to perform follow-up studies or therapeutic monitoring. As described in 3-month-old

380 DMD piglets ${ }^{59}$, we now validated our longitudinal imaging results by proving the increase in

381 muscular fibrosis and increased abundance of ECM proteins (e.g. collagens) even at the age of

382 four weeks.

383 Novel therapeutic approaches leading to an ultrastructural restoration of damaged muscles ${ }^{60}$

384 might therefore be directly visualized using MSOT in future studies. While our findings remain

385 preliminary, our approach starting with experimental tissues and progressing through to first in-

386 patient application supports MSOT-derived collagen detection and quantification as a potential 
387 age-independent imaging biomarker for disease progress monitoring in DMD and suggest the 388 potential for further applications.

389 


\section{Acknowledgements}

391 F.K. acknowledges founding from Else Kröner-Fresenius-Stiftung (Else Kröner-Memorial-

392 Stipendium, 2018_EKMS.03). A.P.R. received support from the ELAN Fond at the University

393 Hospital of the Friedrich-Alexander-Universität (FAU) Erlangen-Nürnberg. F.K. and A.P.R.

394 acknowledge support by the Interdisciplinary Center for Clinical Research (IZKF) at the

395 University Hospital of the Friedrich-Alexander-Universität (FAU) Erlangen-Nürnberg. A.M.N.

396 acknowledges funding from Johannes and Frieda Marohn Foundation. MJW received support

397 from the Graduate School in Advanced Optical Technologies of the Friedrich-Alexander-

398 Universität (FAU) Erlangen-Nürnberg. MFN acknowledges funding from the Emerging Fields

399 Initiative (EFI) of the Friedrich-Alexander-Universität (FAU) Erlangen-Nürnberg. M.J.W and

400 MFN acknowledge founding from German Research Foundation (FOR2438, TRR241). E.W.

401 acknowledges funding from Else Kröner-Fresenius Foundation (2015_180; 2018_T20),

402 Bayerische Forschungsstiftung (AZ 802/08) and German Research Foundation (TRR127).

403 M.F.N., M.J.W., F.K. received funding from the European Union’s Horizon 2020 research and

404 innovation programme under grant agreement No 830965. We thank the Imaging Science

405 Institute (Erlangen, Germany) for providing us with the measurement time at the 3 T MRI

406 system. The present work was performed in partial fulfillment of the requirements for obtaining

407 the degree „Dr. med. vet.“ (LMF). We thank the patients and healthy volunteers who committed

408 their time and effort. We grateful acknowledge our physiotherapists Jutta Tolks, Martina Müller-

409 Allissat and Patricia Poepperl for excellent assistance and physical testing of the participants. We

410 thank the administrative staff at the Center for Social Pediatrics and the Center for Rare

411 Neuromuscular Diseases within the Center for Rare Disease at the University Hospital Erlangen

412 with a special thanks to Stephanie Schuessler, M.D. for help during patient recruitment, Gudrun 
413 Boie and Ida Allabauer for performing histological techniques, Christina Blechinger for

414 exceptional help during animal husbandry and histological preparations, Benjamin Marty, PhD,

415 for fruitful discussions about MRI in muscular diseases. Guy Poland, D.V.M., for language

416 editing and Matthias Englbrecht, PhD, for statistical review of the manuscript.

\section{Author contributions}

419 R.T. and F.K. conceived the idea of the study. Phantom imaging was performed by A.P.R., 420 A.L.W. and F.K. A.P.R., R.T., M.J.W., and F.K. designed the study and recruited the pediatric 421 participants. R.T., M.J.W. and F.K. were the principal investigators of the pediatric study.

422 Ultrasound imaging was performed by J.J. M.Q. provided device support. The animal model was 423 designed by E.W. and N.K. The animal studies were designed by A.P.R., L.M.F., E.W., M.J.W. 424 and F.K. E.W. was the principal investigator of the animal study and A.P.R., L.M.F., A.L.W., 425 and F.K. performed the imaging studies. Pediatric MSOT imaging was performed by A.P.R. and 426 F.K. Human MRI imaging was performed and analyzed by T.G., A.M.N., R.H., A.P.R. and M.U. 427 Ex vivo tissue analyses were performed by L.M.F., E.K., A.P.R. and F.K. T.F. and F.F.

428 performed mass spectrometry. Data collection was completed and analyzed by A.P.R. and F.K. 429 A.P.R., L.M.F., M.F.N., E.W., T.G., W.R., J.W., M.J.W., and F.K. interpreted the data. A.P.R. 430 and F.K. wrote the first draft of the manuscript. The manuscript was critically reviewed by all 431 authors.

\section{Competing interests}

434 A.P.R., M.J.W., F.K. are co-inventors together with iThera Medical GmbH, Germany on an EU 435 patent application (EP 19163 304.9) relating to a device and a method for analyzing 
436 optoacoustic data, a optoacoustic system and a computer program. A.P.R., M.J.W., and F.K.

437 received travel support by iThera Medical GmbH, Germany. F.K. reports lecture fees from

438 Siemens Healthcare GmbH outside the submitted work. All other authors declare no competing

439 interests.

440 
1. Mendell, J.R. \& Lloyd-Puryear, M. Report of MDA muscle disease symposium on newborn screening for Duchenne muscular dystrophy. Muscle Nerve 48, 21-26 (2013).

2. $\quad$ Mercuri, E. \& Muntoni, F. Muscular dystrophies. Lancet 381, 845-860 (2013).

3. Hoffman, E.P., Brown, R.H., Jr. \& Kunkel, L.M. Dystrophin: the protein product of the Duchenne muscular dystrophy locus. Cell 51, 919-928 (1987).

4. $\quad$ Klingler, W., Jurkat-Rott, K., Lehmann-Horn, F. \& Schleip, R. The role of fibrosis in Duchenne muscular dystrophy. Acta Myol 31, 184-195 (2012).

5. Bushby, K., et al. Ataluren treatment of patients with nonsense mutation dystrophinopathy. Muscle Nerve 50, 477-487 (2014).

6. McDonald, C.M., et al. Ataluren in patients with nonsense mutation Duchenne muscular dystrophy (ACT DMD): a multicentre, randomised, double-blind, placebo-controlled, phase 3 trial. Lancet 390, 1489-1498 (2017).

7. Mendell, J.R., et al. Longitudinal effect of eteplirsen versus historical control on ambulation in Duchenne muscular dystrophy. Ann Neurol 79, 257-271 (2016).

8. $\quad$ Fairclough, R.J., Wood, M.J. \& Davies, K.E. Therapy for Duchenne muscular dystrophy: renewed optimism from genetic approaches. Nat Rev Genet 14, 373-378 (2013).

9. Klymiuk, N., et al. Tailored Pig Models for Preclinical Efficacy and Safety Testing of Targeted Therapies. Toxicol Pathol 44, 346-357 (2016).

10. Brooke, M.H., et al. Clinical investigation in Duchenne dystrophy: 2. Determination of the "power" of therapeutic trials based on the natural history. Muscle Nerve 6, 91-103 (1983).

11. Escolar, D.M., et al. Randomized, blinded trial of weekend vs daily prednisone in Duchenne muscular dystrophy. Neurology 77, 444-452 (2011).

12. Mayhew, J.E., et al. Reliable surrogate outcome measures in multicenter clinical trials of Duchenne muscular dystrophy. Muscle Nerve 35, 36-42 (2007).

13. Straub, V., et al. Stakeholder cooperation to overcome challenges in orphan medicine development: the example of Duchenne muscular dystrophy. Lancet Neurol 15, 882-890 (2016).

14. Willcocks, R.J., et al. Multicenter prospective longitudinal study of magnetic resonance biomarkers in a large duchenne muscular dystrophy cohort. Ann Neurol 79, 535-547 (2016).

15. Weber, M.A., et al. Permanent muscular sodium overload and persistent muscle edema in Duchenne muscular dystrophy: a possible contributor of progressive muscle degeneration. $J$ Neurol 259, 2385-2392 (2012).

16. Wary, C., et al. Quantitative NMRI and NMRS identify augmented disease progression after loss of ambulation in forearms of boys with Duchenne muscular dystrophy. NMR Biomed 28, 1150-1162 (2015).

17. Hooijmans, M.T., et al. Elevated phosphodiester and T2 levels can be measured in the absence of fat infiltration in Duchenne muscular dystrophy patients. NMR Biomed 30(2017).

18. Gerhalter, T., et al. (23) Na MRI Depicts Early Changes in Ion Homeostasis in Skeletal Muscle Tissue of Patients With Duchenne Muscular Dystrophy. J Magn Reson Imaging (2019).

19. Forbes, S.C., et al. Skeletal muscles of ambulant children with Duchenne muscular dystrophy: validation of multicenter study of evaluation with MR imaging and MR spectroscopy. Radiology 269, 198-207 (2013).

20. McDonald, C.M., et al. The 6-minute walk test in Duchenne/Becker muscular dystrophy: longitudinal observations. Muscle Nerve 42, 966-974 (2010).

21. McDonald, C.M., et al. The 6-minute walk test as a new outcome measure in Duchenne muscular dystrophy. Muscle Nerve 41, 500-510 (2010).

22. Szigyarto, C.A. \& Spitali, P. Biomarkers of Duchenne muscular dystrophy: current findings. Degener Neurol Neuromuscul Dis 8, 1-13 (2018).

23. Ntziachristos, V. \& Razansky, D. Molecular imaging by means of multispectral optoacoustic tomography (MSOT). Chem Rev 110, 2783-2794 (2010).

24. Taruttis, A. \& Ntziachristos, V. Advances in real-time multispectral optoacoustic imaging and its applications. Nat Photonics 9, 219-227 (2015).

25. Stoffels, I., et al. Metastatic status of sentinel lymph nodes in melanoma determined noninvasively with multispectral optoacoustic imaging. Sci Transl Med 7, 317ra199 (2015). 
26. Diot, G., et al. Multispectral Optoacoustic Tomography (MSOT) of Human Breast Cancer. Clin Cancer Res 23, 6912-6922 (2017).

27. Becker, A., et al. Multispectral optoacoustic tomography of the human breast: characterisation of healthy tissue and malignant lesions using a hybrid ultrasound-optoacoustic approach. Eur Radiol 28, 602-609 (2018).

28. Rey-Barroso, L., et al. Visible and Extended Near-Infrared Multispectral Imaging for Skin Cancer Diagnosis. Sensors (Basel) 18(2018).

29. Waldner, M.J., et al. Multispectral Optoacoustic Tomography in Crohn's Disease: Noninvasive Imaging of Disease Activity. Gastroenterology 151, 238-240 (2016).

30. Knieling, F., et al. Multispectral Optoacoustic Tomography for Assessment of Crohn's Disease Activity. $N$ Engl J Med 376, 1292-1294 (2017).

31. Sekar, S.K., et al. Diffuse optical characterization of collagen absorption from 500 to $1700 \mathrm{~nm}$. J Biomed Opt 22, 15006 (2017).

32. Cao, Q., Zhegalova, N.G., Wang, S.T., Akers, W.J. \& Berezin, M.Y. Multispectral imaging in the extended near-infrared window based on endogenous chromophores. J Biomed Opt 18, 101318 (2013).

33. Scholkmann, F., et al. A review on continuous wave functional near-infrared spectroscopy and imaging instrumentation and methodology. Neuroimage 85 Pt 1, 6-27 (2014).

34. Weber, J., Beard, P.C. \& Bohndiek, S.E. Contrast agents for molecular photoacoustic imaging. Nat Methods 13, 639-650 (2016).

35. Taroni, P., et al. Non-invasive optical estimate of tissue composition to differentiate malignant from benign breast lesions: A pilot study. Sci Rep 7, 40683 (2017).

36. Ford, S.J., et al. Structural and Functional Analysis of Intact Hair Follicles and Pilosebaceous Units by Volumetric Multispectral Optoacoustic Tomography. J Invest Dermatol 136, 753-761 (2016).

37. Klymiuk, N., et al. Dystrophin-deficient pigs provide new insights into the hierarchy of physiological derangements of dystrophic muscle. Hum Mol Genet 22, 4368-4382 (2013).

38. Zaidman, C.M., Malkus, E.C. \& Connolly, A.M. Muscle ultrasound quantifies disease progression over time in infants and young boys with duchenne muscular dystrophy. Muscle Nerve 52, 334-338 (2015).

39. Weng, W.C., et al. Evaluation of muscular changes by ultrasound Nakagami imaging in Duchenne muscular dystrophy. Sci Rep 7, 4429 (2017).

40. Bonati, U., et al. Quantitative muscle MRI: A powerful surrogate outcome measure in Duchenne muscular dystrophy. Neuromuscul Disord 25, 679-685 (2015).

41. Barnard, A.M., et al. Skeletal muscle magnetic resonance biomarkers correlate with function and sentinel events in Duchenne muscular dystrophy. PLoS One 13, e0194283 (2018).

42. Glemser, P.A., et al. (23)Na MRI and myometry to compare eplerenone vs. glucocorticoid treatment in Duchenne dystrophy. Acta Myol 36, 2-13 (2017).

43. Arpan, I., et al. Examination of effects of corticosteroids on skeletal muscles of boys with DMD using MRI and MRS. Neurology 83, 974-980 (2014).

44. Weber, M.A., Nagel, A.M., Jurkat-Rott, K. \& Lehmann-Horn, F. Sodium (23Na) MRI detects elevated muscular sodium concentration in Duchenne muscular dystrophy. Neurology 77, 2017-2024 (2011).

45. Juras, V., et al. Histological correlation of $7 \mathrm{~T}$ multi-parametric MRI performed in ex-vivo Achilles tendon. Eur J Radiol 82, 740-744 (2013).

46. Frantz, C., Stewart, K.M. \& Weaver, V.M. The extracellular matrix at a glance. J Cell Sci 123, 4195-4200 (2010).

47. Serrano, A.L. \& Munoz-Canoves, P. Regulation and dysregulation of fibrosis in skeletal muscle. Exp Cell Res 316, 3050-3058 (2010).

48. Negroni, E., et al. Glycosaminoglycan modifications in Duchenne muscular dystrophy: specific remodeling of chondroitin sulfate/dermatan sulfate. J Neuropathol Exp Neurol 73, 789-797 (2014).

49. Desguerre, I., et al. Endomysial fibrosis in Duchenne muscular dystrophy: a marker of poor outcome associated with macrophage alternative activation. J Neuropathol Exp Neurol 68, 762-773 (2009).

50. Bettica, P., et al. Histological effects of givinostat in boys with Duchenne muscular dystrophy. Neuromuscular disorders : NMD 26, 643-649 (2016).

51. Hooijmans, M.T., et al. Non-uniform muscle fat replacement along the proximodistal axis in Duchenne muscular dystrophy. Neuromuscul Disord 27, 458-464 (2017).

52. Chrzanowski, S.M., et al. Multi-slice MRI reveals heterogeneity in disease distribution along the length of muscle in Duchenne muscular dystrophy. Acta Myol 36, 151-162 (2017). 
$550 \quad 53 . \quad$ Emery, A.E. Muscle histology and creatine kinase levels in the foetus in Duchenne muscular dystrophy.

54. Toop, J. \& Emery, A.E. Muscle histology in fetuses at risk for Duchenne muscular dystrophy. Clin Genet 5, 230-233 (1974).

55. Peverelli, L., et al. Histologic muscular history in steroid-treated and untreated patients with Duchenne dystrophy. Neurology 85, 1886-1893 (2015).

56. Bell, C.D. \& Conen, P.E. Histopathological changes in Duchenne muscular dystrophy. J Neurol Sci 7, 529544 (1968).

57. Joseph, J., et al. Evaluation of Precision in Optoacoustic Tomography for Preclinical Imaging in Living Subjects. J Nucl Med 58, 807-814 (2017).

58. Helfen, A., et al. Multispectral Optoacoustic Tomography: Intra- and Interobserver Variability Using a Clinical Hybrid Approach. J Clin Med 8(2019).

59. Frohlich, T., et al. Progressive muscle proteome changes in a clinically relevant pig model of Duchenne muscular dystrophy. Scientific reports 6, 33362 (2016).

60. Min, Y.L., et al. CRISPR-Cas9 corrects Duchenne muscular dystrophy exon 44 deletion mutations in mice and human cells. Sci Adv 5, eaav4324 (2019). 
Figure 1 - exNIR MSOT can detect collagen.

568 a: Schematic of the concepts underlying MSOT. MSOT is based on the photoacoustic effect:

569 pulsed multi-wavelength laser light illuminates tissues down to $3 \mathrm{~cm}$ in depth. Absorption of the

570 light by different chromophores (e.g., hemoglobin, lipids, melanin, collagens) leads to ultrasound

571 vibrations, resulting in pressure waves that can be detected and reconstructed into images.

572 Different chromophores can be separated by spectral unmixing based on the specific absorption

573 and reflection properties of the emitted light. RUCT, reflection ultrasound computed

574 tomography. MSP, multispectral processing.

575 b: Solutions of purified human collagens (I, III, and IV), hemoglobin and lipids, imaged every 5 $576 \mathrm{~nm}$ from $660-1200 \mathrm{~nm}$. Left, demonstration of the ability of MSOT to separate collagens (I, III, 577 and IV) from hemoglobin and lipids by spectral unmixing. Right, quantification of the separated 578 optoacoustic spectra for each chromophore. A representative result from two independent 579 experiments with similar results is shown. Scale bar, $5 \mathrm{~mm}$.

580 c: Left, example of an ex vivo porcine tendon in a custom made phantom (top) and the 581 corresponding MSOT/RUCT merged images image (bottom) showing the MSOT signal for 582 collagen (turquoise) as color-coded map overlaid on the gray-scaled RUCT image. The region of 583 interest (ROIs, white ellipse) is outlined in the RUCT. Right, normalized optoacoustic spectra of 584 collagens of the ex vivo tendon, human purified collagen I, and a collagen spectrum from the 585 literature ${ }^{31}$. A representative result from two independent experiments with similar results is 586 shown. Scale bar, $2 \mathrm{~cm}$.

587 d: Examples of in vivo human tendon imaging by NIR/exNIR MSOT. MSOT/RUCT merged 588 images show signals for de-/oxygenated hemoglobin (red/blue), lipids (yellow) and collagen 589 (turquoise) as color-coded maps overlaid on the gray-scaled RUCT image. The MSOT collagen 
590 signal was mainly found within the tendon, whereas lipids and hemoglobin signals mainly

591 appeared beneath the tendon or in surrounding tissues. NIR, near-infrared range (660 - $900 \mathrm{~nm}$ ).

592 exNIR, extended near-infrared range $(900-1100 \mathrm{~nm})$. A representative result from two

593 independent experiments with similar results is shown. Scale bar, $5 \mathrm{~mm}$.

594 


\section{Figure 2 - in vivo 2D MSOT imaging of newborn piglets}

a: Representative images of skeletal muscle from healthy (WT) (upper row) and DMD (bottom row) piglets. Regions of interest (yellow boxes) are determined in the RUCT images. Qualitative differences of spectrally unmixed optoacoustic collagen signals between WT and DMD piglets are shown in turquoise. The merged MSOT/RUCT image visualizes the collagen distribution

602 within the muscle and the ROI, respectively. A representative result for a WT and a DMD from $n$ $603=58$ independent muscle regions $(\mathrm{n}=34 \mathrm{WT} / \mathrm{n}=24 \mathrm{DMD})$ in $\mathrm{n}=17$ biologically independent 604 animals ( $\mathrm{n}=10 \mathrm{WT} / \mathrm{n}=7$ DMD piglets) from $\mathrm{n}=3$ litters with similar results is shown. Scale 605 bar, $5 \mathrm{~mm}$.

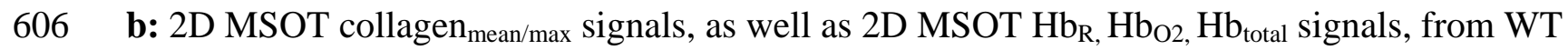
607 and DMD piglets. Each filled circle represents one MSOT signal per independent muscle region 608 (upper row) or the mean MSOT signal per independent animal (bottom row). $\mathrm{n}=58$ independent 609 muscle regions $(\mathrm{n}=34 \mathrm{WT} / \mathrm{n}=24 \mathrm{DMD})$ in $\mathrm{n}=17$ biologically independent animals $(\mathrm{n}=10$ $610 \mathrm{WT} / \mathrm{n}=7$ DMD piglets) from $\mathrm{n}=3$ litters are shown.

611 c: The area under the ROC curve (AUC) and 95\% confidence interval for distinguishing between 612 muscles from DMD and WT piglets, as calculated using unmixed MSOT collagen signals

613 (collagen $n_{\text {mean }}$ and collagen ${ }_{\max }$ ) for $\mathrm{n}=58$ independent muscle regions $(\mathrm{n}=34 \mathrm{WT} / \mathrm{n}=24 \mathrm{DMD})$

614 in $\mathrm{n}=17$ biologically independent animals ( $\mathrm{n}=10 \mathrm{WT} / \mathrm{n}=7$ DMD piglets) from $\mathrm{n}=3$ litters are 615 shown.

616 d: Representative hematoxylin \& eosin (H\&E), Masson’s Trichrome (TriC), Sirius Red (SirR), 617 and dystrophin (Dys1) immunohistochemistry staining from imaged WT (upper row) and DMD 618 (bottom row) piglet musculature. DMD piglet musculature shows disrupted muscular structure 619 (H\&E staining), increased collagen content (turquoise/red), and lacking dystrophin expression 
620 (brown). Black boxes represent areas that are shown at higher magnification in the insets. A

621 representative result for a WT and a DMD muscle from $n=29$ independent muscle specimens ( $\mathrm{n}$

$622=17 \mathrm{WT} / \mathrm{n}=12 \mathrm{DMD})$ from $\mathrm{n}=9$ biologically independent animals $(\mathrm{n}=6 \mathrm{WT} / \mathrm{n}=3 \mathrm{DMD}$

623 piglets) from $n=3$ litters with similar results is shown. Scale bars, $100 \mu \mathrm{m}$ in main micrographs

624 and $50 \mu \mathrm{m}$ in insets.

625 e-f: Quantitative tissue analyses, showing positive-stained collagen area as assessed by TriC (e)

626 and SirR (f) staining. Correlations between the positive-stained collagen areas and the MSOT

627 collagen $_{\text {mean }}$ signal are also shown. $\mathrm{n}=29$ independent muscle specimens $(\mathrm{n}=17 \mathrm{WT} / \mathrm{n}=12$

628 DMD) from $n=9$ biologically independent animals ( $n=6 \mathrm{WT} / \mathrm{n}=3$ DMD piglets) from $\mathrm{n}=3$

629 litters.

630 g: Quantification of total collagen abundance in WT and DMD piglets (mean values of $46.83 \mu \mathrm{g}$

631 collagen/mg protein and $64.95 \mu$ g collagen/mg protein, respectively). The correlation between

632 collagen quantification and the MSOT collagen ${ }_{\text {mean }}$ signal is also shown. $\mathrm{n}=30$ independent

633 muscle specimens $(\mathrm{n}=18 \mathrm{WT} / \mathrm{n}=12$ DMD) from $\mathrm{n}=9$ biologically independent animals $(\mathrm{n}=6$

$634 \mathrm{WT} / \mathrm{n}=3$ DMD piglets) from $\mathrm{n}=3$ litters.

635 b, e-g: Two-tailed independent samples t-tests (with Welch’s correction in cases of unequal

636 variances) was used for statistical analysis. If the assumption of normal distribution was violated,

637 a Mann-Whitney U-test was used. P values $\leq 0.05$ were considered statistically significant. No

638 adjustment for multiple comparison was applied, due to only one single comparison per

639 parameter. Confidence intervals $(95 \% \mathrm{CI})$, effect size $\left(\mathrm{R}^{2}\right)$, coefficients $(\mathrm{t}(\mathrm{df}) / \mathrm{U})$ and exact $\mathrm{p}$

640 values are noted in the main text and/or Supplementary Tables. Data are shown as mean \pm SD.

641 e-g: Correlations are given by Spearman correlation coefficient $\left(\mathrm{r}_{\mathrm{s}}\right)$. Two-tailed test. Linear

642 regression lines are in black. P values $\leq 0.05$ were considered statistically significant. 


\section{Figure 3 - in vivo 2D MSOT imaging of healthy volunteers and DMD patients}

644 a: Example of real-time imaging of a 3-year old healthy volunteer (HV) using the 2D MSOT detector 645 probe.

646 b: Representative MSOT images of transversal scans from four anatomical regions of a 7-year-old HV 647 (left panels) as compared to those of a 5-year-old with DMD (right panels). RUCT images, obtained for anatomical guidance during the examination, are also shown. MSOT/RUCT merged images show MSOT signals for hemoglobin (red) and collagen (turquoise) as color-coded maps overlaid on the gray-scaled RUCT image. Yellow boxes indicate regions of interest that were used for signal quantification. A

651 representative result for a HV and a DMD from $n=159$ independent muscle regions $(n=80$ HV/n $=79$

652 DMD) in $n=20$ biologically independent subjects ( $n=10 \mathrm{HV} / \mathrm{n}=10 \mathrm{DMD}$ patients) with similar results 653 is shown. Scale bar, $5 \mathrm{~mm}$.

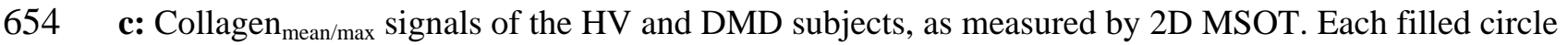
655 represents one MSOT signal per independent muscle region (upper row) or the mean MSOT signal per 656 independent subject (bottom row). Two-tailed dependent samples t-tests (matched for age) was used for 657 statistical analysis. If the assumption of normal distribution was violated Wilcoxon signed-rank tests was 658 used. Bonferroni-Holm adjustment was used to control type I error. Confidence intervals (95\%CI), effect 659 sizes $\left(\mathrm{R}^{2}\right)$, coefficients $\left(\mathrm{t}(\mathrm{df}) / \mathrm{W}^{-}\right)$and $\mathrm{p}$ values are noted in the main text and Supplementary Tables. $\mathrm{p}$ 660 values $\leq 0.05$ were considered statistically significant. $\mathrm{n}=158$ matched independent muscle regions $(\mathrm{n}=$ $66179 \mathrm{HV} / \mathrm{n}=79 \mathrm{DMD}$ ) of $\mathrm{n}=20$ biologically independent subjects ( $\mathrm{n}=10 \mathrm{HV} / \mathrm{n}=10 \mathrm{DMD}$ patients). Data 662 are shown as mean \pm SD.

663 d: ROC curves for distinguishing between DMD and HV muscles using unmixed MSOT collagen

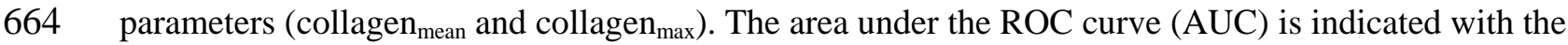
$66595 \%$ confidence interval for distinguishing between muscles from HV and DMD patients, as calculated 666 using unmixed MSOT collagen parameters (collagen ${ }_{\text {mean }}$ and collagen ${ }_{\max }$ ) for $\mathrm{n}=159$ independent muscle 
667 regions $(\mathrm{n}=80 \mathrm{HV} / \mathrm{n}=79 \mathrm{DMD})$ in $\mathrm{n}=20$ biologically independent subjects $(\mathrm{n}=10 \mathrm{HV} / \mathrm{n}=10 \mathrm{DMD}$ 668 patients).

669

670 


\section{Figure 4 - in vivo 3D MSOT imaging of healthy volunteers and DMD patients}

672 a: Cartoon of the handheld 3D hemispherical MSOT detector probe (8 MHz center frequency). Using the

673 NIR (660-900nm) and exNIR (900-1100nm) spectrum of light (660-1100nm), different absorbers, such as

674 collagens, can be detected by their specific acoustic spectra. 3D imaging allows volumetric acquisition

675 and quantification of tissue components.

676 b: 3D MSOT images of the same two boys from Fig. 3b are shown. Top row, 7-year-old HV; bottom

677 row, 5-year-old DMD subject. Maximum projection images of the gastrocnemius muscle in two axes (XZ

678 and YZ) and a 3D volumetric (volume) area are depicted with color-coded maps showing $\mathrm{Hb}_{\text {total }}($ red),

679 collagen $_{\text {mean }}$ (turquoise) and lipid (yellow). Scale bar, $5 \mathrm{~mm}$. A representative result for a HV and a DMD

680 from $n=160$ independent muscle regions $(n=80 \mathrm{HV} / \mathrm{n}=80$ DMD) in $\mathrm{n}=20$ biologically independent

681 subjects ( $\mathrm{n}=10 \mathrm{HV}$ and $\mathrm{n}=10$ DMD patients) with similar results is shown.

682 c: Quantification of 3D MSOT signals $\left(\mathrm{Hb}_{\mathrm{R}}=\right.$ deoxygenated hemoglobin, $\mathrm{HbO}_{2}=$ oxygenated

683 hemoglobin, $\mathrm{Hb}_{\text {total }}=$ total hemoglobin, collagen ${ }_{\text {mean, }}$, collagen $\mathrm{max}_{\mathrm{m}}$ ). Each filled circle represents one MSOT

684 signal per independent muscle region (upper row) or the mean MSOT signal per independent subject

685 (bottom row).

686 Two-tailed dependent samples t-tests (matched for age) was used for statistical analysis. If the assumption

687 of normal distribution was violated Wilcoxon signed-rank tests was used. Bonferroni-Holm adjustment

688 was used to control type I error. Confidence intervals (95\%CI), effect sizes $\left(\mathrm{R}^{2}\right)$, coefficients (t(df)/ $\left.\mathrm{W}^{-}\right)$

689 and $\mathrm{p}$ values are noted in the main text and/or Supplementary Tables. $\mathrm{p}$ values $\leq 0.05$ were considered

690 statistically significant. $n=160$ matched independent muscle regions $(n=80 \mathrm{HV} / \mathrm{n}=80 \mathrm{DMD})$ in $n=20$

691 biologically independent subjects ( $n=10 \mathrm{HV}$ and $\mathrm{n}=10 \mathrm{DMD}$ patients). Data are shown as mean \pm SD.

692 d: ROC curves for distinguishing between DMD and HV muscles using unmixed MSOT collagen

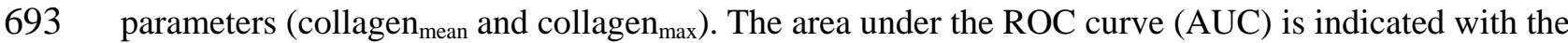

$69495 \%$ confidence interval. $\mathrm{n}=160$ independent muscle regions $(\mathrm{n}=80 \mathrm{HV} / \mathrm{n}=80 \mathrm{DMD})$ in $\mathrm{n}=20$

695 biologically independent subjects ( $\mathrm{n}=10 \mathrm{HV}$ and $\mathrm{n}=10$ DMD patients). 


\section{Figure 5 - Correlation of MSOT imaging and clinical standard assessments}

698 a: Correlation matrix of physical examinations, age, and mean MSOT collagen mean/max $_{\text {signals. }}$

699 Correlations are indicated as highly positive (dark blue), highly negative (dark red,) or white (non-

700 significant) and Spearman correlation coefficient is given in numbers. MRC $=$ Medical Research Council

701 (MRC) strength grading system, UE = upper extremity, LE = lower extremity, prox. = proximal, dist. =

702 distal, 6-MWT $=6$-minute-walk-test, sit to stand= sit to stand test, and 4/8 stairs $=4 / 8$ stairs climb.

703 Correlations are given by Spearman correlation coefficient $\left(\mathrm{r}_{\mathrm{s}}\right)$. Two-tailed test. Two-tailed test.

704 Bonferroni-Holm adjustment was used to control type I error, due to four comparisons (2D/3D

705 collagen $_{\operatorname{mean} / \max }$ ) per parameter (e.g. 6-MWT). P values $\leq 0.05$ were considered statistically significant. $n$

$706=20$ biologically independent subjects ( $\mathrm{n}=10 \mathrm{HV} / \mathrm{n}=10 \mathrm{DMD}$ patients). Detailed p-values and numbers

707 (n) are presented in the Supplementary Table 6.

708 b: Representative MRI images (water $\mathrm{T}_{2}$ map, fat fraction (FF) map, total tissue sodium concentration

709 (TSC) map, and intracellular-weighted ${ }^{23} \mathrm{Na}$ signal (ICwS)) map of a 7-year old DMD subject, compared

710 to corresponding transversal 2D MSOT images (dashed line is drawn around the gastrocnemius muscle

711 region). A representative result for a DMD patient from $\mathrm{n}=5$ biologically independent subjects with

712 similar results is shown. Scale bars, $1 \mathrm{~cm}$ in MRI images and $5 \mathrm{~mm}$ in MSOT images. 


\section{Figure 6 - MSOT quantitatively visualizes early-stage disease progression over time}

714 a: Study outline for longitudinal monitoring of DMD piglets. Imaging was performed at weeks 1, 2, 3,

715 and 4 of life. All piglets were sacrificed after week 4, and tissue specimens were harvested.

716 b: Survival of WT and DMD piglets over the time course of the experiment. Kaplan-Meier curve of WT

717 piglets $(n=6)$ and DMD piglets $(n=5)$. Circles represent censored subjects or unexpected death of

718 subjects.

719 c: Schematic indicating how standardized palpable landmarks were used to locate muscles in piglets prior

720 to imaging, ensuring repeatability of detector placement over time. Exemplary landmarks and skinned

721 muscles are shown. Scale bar, $10 \mathrm{~cm}$.

722 d: Representative 3D MSOT images of one DMD piglet over 4 weeks. Color-coded maps show $\mathrm{Hb}_{\text {total }}$

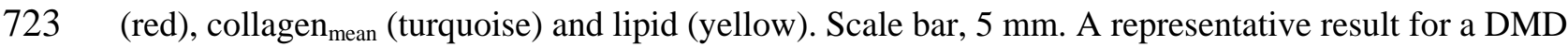

724 piglet from $n=5$ biologically independent animals $(n=3 \mathrm{WT} / \mathrm{n}=2$ DMD piglets $)$ from $\mathrm{n}=2$ litters is

725 shown.

726 e: Quantification of 2D and 3D MSOT collagen ${ }_{\text {mean }}$ signals in WT and DMD piglet muscles over time. In

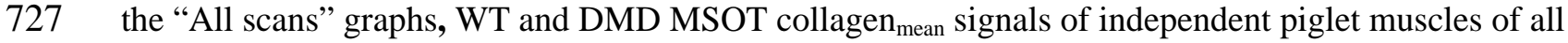

728 animals were compared with each other at weeks 1, 2, 3, and 4 of age. Each filled circle represents one

729 MSOT signal per independent muscle region $(\mathrm{n}=24 \mathrm{WT} / \mathrm{n}=20 \mathrm{DMD})$. Two-tailed independent samples

730 t-tests (with Welch's correction in cases of unequal variances) was used for statistical analysis. If the

731 assumption of normal distribution was violated, a Mann-Whitney U-test was used. P values $\leq 0.05$ were

732 considered statistically significant. Bonferroni-Holm adjustment was used to control type I error, due to

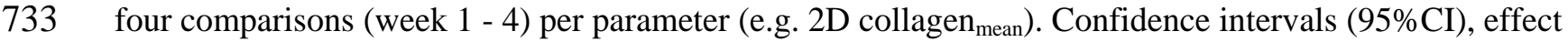

734 size $\left(\mathrm{R}^{2}\right)$, coefficients (t(df)/U) and exact p values are noted in the main text and/or Supplementary

735 Tables. Data are shown as mean \pm SD. $n=44$ independent muscle regions $(n=24$ WT/n $=20$ DMD) in $n$

$736=11$ biologically independent animals $(\mathrm{n}=6 \mathrm{WT} / \mathrm{n}=5$ DMD piglets) from $\mathrm{n}=2$ litters are shown.

737 In the "Completed scans” graphs, WT and DMD MSOT collagen ${ }_{\text {mean }}$ signals of independent piglet

738 muscles of surviving animals were compared with each other at weeks 1, 2, 3, and 4 of age. Each filled 
circle/square represents the mean \pm SD MSOT signal of independent muscle regions over the course of the experiment $(\mathrm{n}=12 \mathrm{WT} / \mathrm{n}=8 \mathrm{DMD})$. 2D MSOT parameters were analyzed by post-hoc Tukey’s HSD

741 following a mixed-effects models due to missing values in week 1 (litter 1 ). p values $\leq 0.05$ were

742 considered statistically significant. 3D MSOT collagen parameters were analyzed by Tukey’s honestly

743 significant difference tests following a two-way (mixed design) ANOVA; Data are shown as mean \pm SD.

$744 \mathrm{n}=20$ independent muscle regions $(\mathrm{n}=12 \mathrm{WT} / \mathrm{n}=8 \mathrm{DMD})$ in $\mathrm{n}=5$ biologically independent animals ( $\mathrm{n}$

$745=3 \mathrm{WT} / \mathrm{n}=2 \mathrm{DMD}$ piglets) from $\mathrm{n}=2$ litters are shown.

746 f-g: A representative image of a 4 week old WT and DMD piglet (f) and representative low magnification 747 views of freshly-excised unstained muscle (macroscopy), as well as TriC and SiR stained muscles, from

748 WT and DMD piglets (g). Macroscopic alterations and increased fibrosis are evident in DMD versus WT 749 muscle TriC and SirR stainings. Scale bars, $1 \mathrm{~cm}$ (macroscopy) and $100 \mu \mathrm{m} / 50 \mu \mathrm{m}$ (histology/inserts).

750 h: Quantitation of the positive-stained collagen area of WT and DMD piglet muscles at weeks 1 and 4 of 751 age, as assessed by TriC and SirR staining and collagen abundance. Two-tailed independent samples t752 tests (with Welch's correction in cases of unequal variances) was used for statistical analysis. If the 753 assumption of normal distribution was violated, a Mann-Whitney U-test was used. Bonferroni-Holm 754 adjustment was used to control type I error, due to three comparisons per parameter (e.g. TriC).

755 Confidence intervals (95\%CI), effect sizes $\left(R^{2}\right)$, degrees of freedom $(t(d f) / U)$ and $p$ values are noted in the 756 main text. P values $\leq 0.05$ were considered statistically significant. Data are shown as mean \pm SD. $n=20$ 757 independent muscle specimens $(n=12 \mathrm{WT} / \mathrm{n}=8 \mathrm{DMD})$ from $\mathrm{n}=5$ biologically independent animals $(\mathrm{n}=$ $7583 \mathrm{WT} / \mathrm{n}=2 \mathrm{DMD}$ ) from $\mathrm{n}=2$ litters.

759 i-k:

760 Quantitative proteome analysis of skeletal muscle tissue from 1- and 4-week-old DMD and WT piglets: 761 principal component analysis (PCA) (i), unsupervised hierarchical clustering of LFQ intensity values (j) 762 and volcano plots of $\log 2$ fold changes (k). In $\mathbf{i}$, symbols represent individual muscle samples. In $\mathbf{k}$, the 763 permutation-based FDR significance cutoff $(<0.05)$ is depicted by the black curves. Two-tailed 
764 independent samples t-tests (with Welch’s correction) was used for statistical analysis. No 765 adjustment for multiple comparison was applied, due to reporting as significant by a

766 permutation-based FDR estimation (FDR $<0.05$ ). p values $\leq 0.05$ were considered statistically

767 significant. $\mathrm{n}=16$ independent muscle specimens $(\mathrm{n}=8 \mathrm{WT} / \mathrm{n}=8 \mathrm{DMD})$ from $\mathrm{n}=8$ biologically

768 independent animals $(n=4 \mathrm{WT} / \mathrm{n}=4 \mathrm{DMD})$ from $\mathrm{n}=2$ litters. 


\section{Phantom imaging}

771

772 Plastic placeholders were positioned in a costume made metal mold and filled with a 2\%

773 agarose/1\% lipid-deuterium oxide-gel (Agarose Standard, Roti@garose, Carl Roth GmbH + Co,

774 KG, Karlsruhe, Germany, heavy water [Deuterium oxide], 99.9\%, Sigma-Aldrich Chemie

775 GmbH, Steinheim, Germany, and Lipofundin @ MCT/LCT 20\%, Braun, Melsungen, Germany).

776 Placeholders were replaced by plastic straws filled with following chromophores: purified

777 human collagen type I (Human Type I Atelo-Collagen Solution, 3 mg/mL, VitroCol@, Advanced

778 Biomatrix, San Diego, USA), type III (Human Collagen Solution, Type III, 1 mg/ml ®,

779 Advanced Biomatrix, San Diego, USA), and type IV (Collagen Type IV from human cell

780 culture, 0.3mg/ml, Sigma-Aldrich Chemie GmbH, Steinheim, Germany), anticoagulated

781 (citrated) blood, lipids (rapeseed oil), and water.

782 For tissue imaging, a plastic container was filled one half with 2\% agarose-gel (Z\%Agarose,

783 NEEO ultra-quality, Carl Roth GmbH + Co, KG, Karlsruhe, Germany). The sample was

784 positioned on the surface of the hardened agarose. The gel suppressed reflections on the back

785 wall of the container. For ideal coupling, it was then filled completely with deionized water. All

786 phantoms and ex vivo samples were imaged with a hybrid ultrasound MSOT system (MSOT

787 Acuity Echo prototype imaging system, iThera Medical GmbH, Munich, Germany) described

788 below.

789

790

\section{Porcine DMD model}

791 All animal experiments were performed in accordance with the German Animal Welfare Act and

792 were approved by the responsible animal welfare authority (District Government of Upper 
793 Bavaria, Reference Number 55.2-1-54-2532-163-2014). A heterozygous female carrier pig with

794 a deletion of DMD exon 52 (DMD 552$)$ was established by somatic cell nuclear transfer (SCNT)

795 using primary cells with one modified DMD allele ${ }^{9,37}$. A breeding herd for DMD pigs was

796 founded on heterozygous $D M D^{+/}$sows, generated by inseminating the founder animal with

797 sperm from WT boars (Prüf- und Besamungsstation München Grub e.V., Munich, Germany).

798 For the proof of concept study, male $D M D^{\mathrm{Y} /}$ piglets were selected as study subjects from three

799 different litters, derived from matings of F1 $D M D^{+/-}$pigs with wild-type boars at the Center for

800 Innovative Medical Models (CiMM, LMU Munich, Germany). For the longitudinal porcine

801 studies, male $D M D^{\mathrm{Y} / \text { - }}$ piglets from two different litters were selected from matings of F2 $D M D^{+/-}$

802 pigs as described above.

803

804 Genotype Screening

805 Genotyping of piglets was carried out by polymerase chain reaction (PCR) analysis of DNA

806 isolated from individual tail biopsies. The abundance of the intact (WT) DMD allele was

807 detected by the primer pair $5^{\prime}$-tgc aca atg ctg gag aac ctc a-3' and $5^{\prime}$-gtt ctg gct tct tga ttg ctg g-

$8083^{\prime}$, whereas the mutated ( $\left.D M D \Delta 52\right)$ allele was detected by the primer pair $5^{\prime}$-cag ctg tgc tcg acg

809 ttg tc-3' and 5'-gaa gaa ctc gtc aag aag gcg ata g-3'.

810

\section{MSOT piglet imaging}

812 For the proof-of-concept study, $\mathrm{N}=17$ piglets underwent standardized 2D MSOT-imaging by

813 transversal scans of the shoulder (triceps brachii muscle) and the leg/thigh (biceps femoris

814 muscle) muscles. All animals were scanned within 1 - 3 days after birth. $\mathrm{N}=11$ piglets were

815 included in the longitudinal porcine pilot study. Here, all animals underwent standardized 
transversal, and 3-dimensional imaging of the shoulder and leg/thigh muscles of both sides (Fig.

817 6c and Supplementary appendix Table 21). Imaging was performed in week 1 (day 1-2), week

8182 (day 2-3), week 3 (day 8/9), and week 4 (day 22/23) of life. Only WT piglets were sedated with

819 intranasal application of Midazolam if required from week 2 onwards. DMD piglets were trained

820 by LMF, and no sedation was required during MSOT imaging. All imaging investigators (AR,

821 FK) were blinded to the genotype.

822

823 Histological examination and collagen quantification

824 In the proof of concept study, $\mathrm{N}=9$ animals ( $\mathrm{N}=6 \mathrm{WT}$ and $\mathrm{N}=3$ DMD piglets) were

825 euthanized 3-8 days after birth and representative tissue specimens were taken from the

826 previously imaged anatomical regions. In the longitudinal study, $\mathrm{N}=10$ animals $(\mathrm{N}=5 \mathrm{WT}$ and

$827 \mathrm{~N}=5 \mathrm{DMD}$ piglets) were examined after death; $\mathrm{N}=5$ in week $1(\mathrm{~N}=3 \mathrm{WT}$ and $\mathrm{N}=2 \mathrm{DMD}), \mathrm{N}$

$828=5$ in week $4(\mathrm{~N}=2 \mathrm{WT}$ and $\mathrm{N}=3 \mathrm{DMD})$. One WT was excluded from histological analysis,

829 due to unknown exact time of death.

830 After harvesting, tissue was fixed in a 4\% formaldehyde/phosphate buffered saline solution (Roti

831 Histofix 4\%; Carl Roth GmbH, Germany), and later embedded in paraffin. Sections (4 $\mu m$ thick)

832 were stained with hematoxylin-eosin (H\&E), Sirius Red (SirR), and Masson trichrome (TriC)

833 according to standard laboratory protocols. Dystrophin immunohistochemistry was performed on

834 sections of formalin-fixed, paraffin-embedded samples of the biceps femoris and triceps brachii

835 muscle of a WT and a DMD piglets. Heat-induced antigen retrieval was performed using $10 \mathrm{mM}$

836 citrate buffer (pH 6.0/0.05\% Tween). Primary antibody was mouse monoclonal antibody against

837 DYS1 (Rod domain) (dilution 1:20; overnight at $4^{\circ} \mathrm{C}$; \#NCL-DYS1; Clone: Dy4/6D3; Leica

838 Biosystems), secondary antibody was biotinylated goat anti-mouse IgG (dilution 1:500; 1 hour at 
839 room temperature; \#115-065-146, Jackson ImmunoResearch). Bound antibodies were detected

840 using the Vectastain Elite ABC HRP Kit (\#PK-6100; Vector Laboratories) and 3,3-

841 diaminobenzidine tetrahydrochloride dihydrate (\#SK-4105; Vector Laboratories) as chromogen

842 (brown color). Hemalaun was used as counterstain (blue color).

843 SirR and TriC stained sections were analyzed for their collagen content as following: histological

844 sections were photographed with an AXIO Scope.A1 (Carl Zeiss AG, Germany) using AXIO

845 Vs40 software (Version 4.8.10, Carl Zeiss Imaging Solutions GmbH) with 10-fold

846 magnification. TIF files (1388x1040 pixels) were exported and analyzed using FIJI software

847 (Version 2.0.0 or newer, available at https://fiji.sc) ${ }^{61}$. Then, the images were split in three

848 channels (red, blue, and green). For collagen quantification in TriC, the red channel was used

849 and for SirR staining the green channel was used. The respective positive stained tissue was

850 calculated as a fraction from the whole images.

851

852 Collagen and protein assays

853 For total collagen (hydroxyproline) and total protein quantification, five paraffin embedded

854 muscle tissue sections (10 $\mu$ m thick) per muscle were analyzed using a Total Collagen Assay

855 (QuickZyme, Biosciences, Leiden, Netherlands) and a Total Protein Assay (QuickZyme,

856 Biosciences, Leiden, Netherlands). According to manufacturer's instructions, the tissue was

857 acidic hydrolyzed (6M HCl, Sigma-Aldrich Chemie GmbH, Steinheim, Germany, overnight at

$\left.85895^{\circ} \mathrm{C}\right)$. The samples for total collagen and total protein content were measured using a

859 microplate reader (ELx808, BioTek, Winooski, US) at $550 \mathrm{~nm}$. The absolute collagen content

860 was derived from the quotient between total collagen and total protein. 


\section{Quantitative proteome analysis of skeletal muscle samples}

863 Frozen tissue samples were cryopulverized using a cryoPREP hammer (Covaris, model no. CP02

864 cryoPREP Impactor) at an impact level of five. Tissue powder was homogenized in lysis buffer

865 (8 M urea/0.4 M NH${ }_{4} \mathrm{HCO}_{3}$ ) by ultrasonification (11 kJ, Sonopuls GM3200 equipped with a

866 BR30 cup booster). Pierce 660 nm Protein Assay (Thermo Fisher Scientific) was used for total

867 protein quantification. $60 \mu \mathrm{g}$ of protein was reduced using dithiothreitol (DTT, final

868 concentration $4 \mathrm{mM}$ ) for $30 \mathrm{~min}$ at $56^{\circ} \mathrm{C}$ and cysteines were alkylated with iodoacetamide (IAA,

869 final concentration $8 \mathrm{mM}$ ) for $30 \mathrm{~min}$ in the dark. Residual IAA was quenched with DTT at a

870 final concentration of $10 \mathrm{mM}$ during $15 \mathrm{~min}$ incubation in the dark. Proteins were digested with

871 Lys-C (Wako Chemicals) at an enzyme/substrate ratio of 1:50 for $4 \mathrm{~h}$ at $37^{\circ} \mathrm{C}$. Samples were

872 diluted with water to $0.8 \mathrm{M}$ urea and digested overnight at $37^{\circ} \mathrm{C}$ with trypsin (1:50; Promega).

873 Nano-LC-MS/MS analysis was conducted on an Ultimate 3000 nano-LC system (Thermo Fisher

874 Scientific) coupled online to a Q Exactive HF-X mass spectrometer (Thermo Fisher Scientific).

$8751.5 \mu \mathrm{g}$ of tryptic peptides were separated at $250 \mathrm{~nL} / \mathrm{min}$ on a $50 \mathrm{~cm}$ column (PepMap RSLC C18,

$87675 \mu \mathrm{m}$ ID, $2 \mu \mathrm{m}$, Thermo Fisher Scientific) from 5\% to 25\% solvent B (0.1\% formic acid in

877 acetonitrile) in $160 \mathrm{~min}$ and from $25 \%$ to $40 \%$ B in $10 \mathrm{~min}$. Spectra were acquired by one full

878 scan (350 to $1600 \mathrm{~m} / \mathrm{z}$ ) with a resolution of 60,000 and up to 15 data-dependent MS/MS scans

879 with a resolution of 15,000. MS raw data were processed using MaxQuant (v. 1.6.7.0) ${ }^{62}$ with

880 FDR $<0.01$ at the peptide and protein level. For protein identification, the NCBI RefSeq Sus

881 scrofa database (v. 3-5-2019) was used. Label-free peptide and protein quantifications were

882 performed by MaxQuant with the normalization feature disabled. Downstream data analysis and

883 visualization was performed using Perseus (v. 1.6.7.0) ${ }^{63}$ and $\mathrm{R}^{64}$ as previously described ${ }^{65}$. 


\section{Study design of pediatric MSOT study}

886 All patients were recruited at the Department of Pediatric Neurology of the University Hospital 887 of Erlangen between June $6^{\text {th }} 2018$ and June $15^{\text {th }} 2018$. Ten boys between 3 and 10 years of age 888 with DMD, confirmed either by genotyping or biopsy, and with preserved walking ability, were 889 enrolled in the study. As controls, ten sex- and age-matched, healthy volunteers (HV) were 890 recruited during the same time period. HV with any pre-existing muscular disorder were 891 excluded. After screening for study inclusion, all participants underwent physical examination, 892 (standard) B-mode ultrasound and MSOT. Data collection included demographic and clinical 893 features such as age, sex, height and weight as well as disease-specific characteristics such as 894 disease duration and current treatment. The study was performed in accordance with the 895 Declaration of Helsinki. Approval from the local ethics committee (Friedrich-Alexander896 University of Erlangen-Nuremberg) was granted and written informed consent was obtained 897 from parents/guardians. The study was registered at clinicaltrials.gov (ID: NCT03490214). For 898 any identifiable images of research participants, written permission to publish was obtained.

\section{Physical examinations}

901 Patients and healthy volunteers underwent physical examination during the course of the study

902 by three well-trained physiotherapists. All participants started with the 6-MWT ${ }^{20,21}$, followed by 903 timed function tests (rise from chair, rise from supine, $10 \mathrm{~m}$ walk/run, 4 stairs climb, 8 stairs 904 climb and sit to stand-test) and manual muscle testing (MRC of 20 muscles/groups including 905 shoulder, arm, hand, fingers, limb, leg, toes, head and trunk (grading 0-5)). ${ }^{10,66-68}$

906 In detail, the respective test was performed and scored as follows: 6-MWT=6-minute-walk-test 907 (measures walking distance within six minutes; range: 0-theoretically infinite; lower distance 
908 represents a higher degree of muscle function loss) ${ }^{20,21}$, sit to stand-test (measures repetitions to

909 rise from chair in one minute; one repetition to theoretically infinite; high number of repetitions

910 indicate a better degree of muscle function and a lower degree of dyspnea; few repetitions

911 indicate a higher degree of muscle function loss and a higher degree dyspnea) adapted from ${ }^{67}$,

912 rise from chair/supine (measures time to rise from chair in seconds; 0.5 seconds to a time limit of

913120 seconds; short time indicates a better degree of muscle function; long time indicates a higher

914 degree of muscle function loss) ${ }^{10,66,68}, 10$-meter walk/run (measures time to run 10 meters in

915 seconds; a few seconds to a time limit of 120 seconds; short time indicates a better degree of

916 muscle function; long time indicates a higher degree of muscle function loss) ${ }^{10,66,68}, 4-/ 8$-stairs

917 climb (measures time to climb 4 stairs in seconds; a few seconds to a time limit of 120 seconds;

918 short time indicates a better degree of muscle function; long time indicates a higher degree of

919 muscle function loss) ${ }^{10,66,68}$, manual muscle testing of 20 muscles using the Medical Research

920 Council (MRC) strength grading system; grade $0-5$ ( $0=$ paralysis to $5=$ full strength). ${ }^{10,66,68}$

921 For statistical analysis the muscles were grouped in 4 anatomical regions (proximal and distal

922 muscles of the upper and lower extremity) and a mean muscle-strength-score was calculated for

923 each region. Duration for physical examination was limited to one hour. Missing data points

924 were excluded from final analysis.

925

926 MRI acquisition and analysis

$927 \mathrm{~N}=5$ DMD patients of our study were simultaneously recruited by the Radiology Department of

928 the University Hospital Erlangen in a separate study for an analysis of ${ }^{23} \mathrm{Na}$ MRI to detect early

929 changes in ion homeostasis in DMD patients between February 2018 to May 2018. The study

930 was approved by the local Ethical Review Board (Number: 250_16 Bc). MR data acquisition 
931 methods have been described previously ${ }^{18}$. Briefly, all participants underwent MRI (Magnetom

932 Skyra, 3 T, Siemens Healthineers, Erlangen, Germany) of their right lower leg. The imaging

933 protocol consisted of a ${ }^{1} \mathrm{H}$ MRI part (using a dedicated 15-channel knee coil (Siemens

934 Healthineers, Germany)) and a ${ }^{23} \mathrm{Na}$ MRI part (using a dedicated 1-channel knee coil (Stark

935 Contrast, Germany). Details of the protocol are displayed in the Supplementary Table 22. Fat

936 fraction (FF) and water T2 maps were obtained from the ${ }^{1} \mathrm{H}$ protocol as well as total sodium

937 concentration (TSC) and intracellular-weighted sodium signal (ICwS) maps from the ${ }^{23} \mathrm{Na}$

938 protocol. ROIs for ${ }^{23} \mathrm{Na} \mathrm{MRI}$ evaluation were drawn on anatomical reference images and

939 interpolated on the sodium maps. For MRI imaging analysis, manually drawn ROIs were placed

940 in the gastrocnemius muscles corresponding to MSOT images (Figure 5b). All MRIs were

941 reviewed by researchers with at least five years of experience (AMN and TG).

942

943 Analysis of ${ }^{23} \mathrm{Na}$ MRI in comparison to MSOT in DMD patients

944 A retrospective comparative analysis of MRI and MSOT was conducted to correlate the

945 respective information. The median of five MRI sections (10mm width, each) was used for the

946 analysis of water $\mathrm{T}_{2}$ maps and FF; TSC and ICwS signals were evaluated from corresponding

947 sections (15mm and 20mm width, respectively). For comparison between MRI and MSOT

948 collagen and fat parameters, transversal 2D MSOT images were used.

949

950 MSOT and ultrasound imaging standardization

951 All patients and healthy controls underwent MSOT and ultrasound imaging of eight predefined

952 anatomical regions (biceps brachii muscle, anterior forearm flexors, rectus femoris muscle and

953 gastrocnemius muscle; both sides). Posture and detector (2D/3D MSOT detectors, ultrasound 
954 detector) placement were standardized and marked to ensure scanning reproducibility for all

955 imaging devices (for details see Supplementary Table 23/Extended Data Fig. 7).

956

957 B-mode ultrasound technical data

958 All anatomical regions were scanned using a single high-end ultrasound system (Logiq L9,

959 General Electric Company, Milwaukee, Wisconsin, USA, Linear probe M12L, 9 MHz) by a

960 single, professional investigator (certification level: DEGUM III). The ultrasound datasets were

961 saved as raw data (DICOM). Echogenicity (hyper-/iso-/hypo-echogenic), Heckmatt-Scale (1-

$9624)^{69}$, muscle-texture (coarse-/medium-/fine-granular) and the distribution pattern (in-

963 /homogeneous/focal) were assessed for each image. ${ }^{\mathbf{7 0}}$ The total investigation time for ultrasound

964 imaging was limited to twenty minutes.

965

966 MSOT technical data

967 All images were obtained with two separate hybrid ultrasound MSOT systems (MSOT Acuity

968 Echo prototype imaging system, iThera Medical GmbH, Munich, Germany), one system was

969 only used for piglets and one only for human imaging. The optoacoustic imaging system is based

970 on a 25Hz pulsed Nd:YAG laser and has two detectors. The 2D concave handheld detector (4

971 MHz center frequency, 256 transducer elements) with a field of view of $30 \mathrm{~mm}$ and spatial

972 resolution of $<150 \mu$ m provides cross sectional images and is combined with a reflective

973 ultrasound computed tomography (RUCT) unit, to anatomically guide optoacoustic imaging

974 during the examination. The 3D hemispherical handheld detector (8 MHz center frequency, 256

975 transducer elements) with a field of view of $15 \mathrm{~mm}$ and a spatial resolution of $100 \mu \mathrm{m}$ provides 
976 isotropic volumetric optoacoustic images. Transparent ultrasound gel (AQUASONIC clear ${ }^{\circledR}$,

977 Parker Laboratories Inc., Fairfield, NJ, USA) was used for coupling between detector and skin.

978 For the phantom and ex vivo experiments, Multispectral optoacoustic tomography signals were

979 acquired from $660 \mathrm{~nm}$ to $1200 \mathrm{~nm}$ in $5 \mathrm{~nm}$ steps. For in vivo imaging, Multispectral optoacoustic

980 tomography signals were acquired at $680,700,730,760,800,850,920,1000,1030,1064$, and

$9811100 \mathrm{~nm}$. A polygonal region of interest was placed just beneath the muscle fascia according to

982 the MSOT-signal.

983

\section{MSOT data analysis}

985 2D imaging data was analyzed using cLabs software (Version 2.66, iThera Medical GmbH, 986 Munich, Germany). The program enables analysis of user-defined MSOT parameters (in 987 arbitrary units, a.u.) and extraction of optoacoustic spectra from the region of interest in the 988 multispectral optoacoustic images. 3D imaging data was analyzed using ViewMSOT software 989 (Version 3.8, iThera Medical GmbH, Munich, Germany). MSOT data were reconstructed with 990 direct backprojection and spatial fluence correction was applied for images acquired with the 2D 991 detector $\left(\mu_{\mathrm{a}}=0.022\right.$ and $\left.\mu_{\mathrm{s}}=10 \mathrm{~cm}^{-1}\right)$. Using spectral unmixing, MSOT values for $\mathrm{Hb}_{\mathrm{R}}, \mathrm{HbO}_{2}$ and 992 collagen were obtained. Collagen unmixing was based on acquired wavelengths of the entire 993 spectral range, whereas $\mathrm{Hb}_{\mathrm{R}}, \mathrm{HbO}_{2}$ signal was calculated from a sub-range $(730 \mathrm{~nm}$ and $850 \mathrm{~nm})$ 994 which is more accurate in unmixing due to increase of water absorptivity at higher wavelengths. 995 Single wavelength of $920 \mathrm{~nm}$ was used to depict lipid signals. To aid interpretation, 3D signals 996 (a.u.) were rescaled: human collagen signals (a.u.) were multiplied by $10^{4}$ and hemoglobin

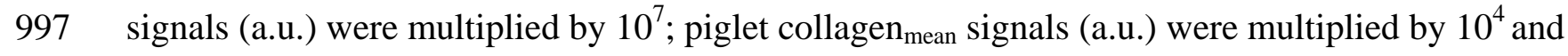
998 collagen $_{\max }$ signals by $10^{3}$. 
1000 Device tolerability

1001 The imaged skin of every participant was visually examined after each scan. Investigated

1002 subjects and parents/guardians were asked about any inconvenience during the imaging

1003 sessions/trials. All reported events were documented.

1004

1005 Statistical analysis

1006 Due to the study design (pilot first-in piglet, first-in pediatric, first longitudinal in vivo use of

1007 MSOT, no former description of collagen signal derived from MSOT in vivo) no sample size

1008 calculation was performed. For piglets, the sample size corresponded to three litters for the proof

1009 of concept, and two litters for the longitudinal porcine pilot trial. Ten Duchenne patients and

1010 healthy volunteers were found to be reasonable and ethically justified for the patient study.

1011 Continuous variables are given as means and standard deviations; categorical variables are

1012 provided as numbers and percentages. Data were tested for normal distribution using Shapiro-

1013 Wilk test prior to inferential analysis. Between piglet group comparisons (DMD vs. WT) of

1014 MSOT parameters and histological results were conducted using independent samples t-tests. In

1015 case of unequal variances in an independent samples t-tests, Welch’s correction was applied. If

1016 the assumption of normal distribution was violated, a Mann-Whitney U-test was chosen for the

1017 independent samples comparison. Correlations are given by Spearman correlation coefficient

$1018\left(\mathrm{r}_{\mathrm{s}}\right)$. Human MSOT parameters were compared between cohorts in a pairwise manner (matched

1019 for age) using dependent samples t-tests. If the assumption of normal distribution was violated

1020 Wilcoxon signed-rank tests was used. We performed receiver operator characteristics (ROC)

1021 analysis between muscles of WT/HV and DMD-piglets/DMD-patients. As gold standard 
1022 histopathology and genotyping were used. Wilcoxon signed-rank tests were used for comparison 1023 of physical examinations. For comparison of the duration of examinations, Mann-Whitney U1024 tests were applied. For longitudinal comparisons of 3D MSOT collagen parameters in the 1025 porcine pilot trial, Tukey’s honestly significant difference tests following a two-way (mixed 1026 design) ANOVA including piglet group and time points were chosen. For longitudinal 1027 comparison of 2D MSOT parameters, post-hoc Tukey's HSD following a mixed-effects models 1028 (fixed effects: piglet group \& time points, random effect: piglet) were used due to missing values 1029 in week 1 (litter 1)). Independent samples t-tests were applied to compare all scans of WT and 1030 DMD piglets at each week. In case of unequal variances in an independent samples t-tests, 1031 Welch's correction was applied. If the assumption of normal distribution was violated, a Mann1032 Whitney U-test was chosen for the independent samples comparison. Quantitative proteome 1033 analysis was performed as described above. All inferential tests were two-tailed, p values $\leq 0.05$ 1034 were considered statistically significant. Bonferroni-Holm adjustment was used to control type I 1035 error. Complete information on inferential test results including test coefficient, p-value, and 1036 effect size are presented in the Supplementary Appendix. All analyses were performed using 1037 GraphPad Prism (Version 7.00 or newer, GraphPad Software, La Jolla, CA, USA).

1039 Statistics and Reproducibility

1040 MSOT signals were derived from averaged video captures (seven frames) obtained in anatomical 1041 independent muscle regions in piglets and patients. All anatomical locations were considered 1042 independent because of the heterogenicity of the underlying disease. In cases of multiple 1043 scans/recordings (e.g. because of piglet/patient movement), a mean of all scans was calculated.

1044 2D transversal and 2D longitudinal MSOT scans at the same anatomical locations were 
1045 considered as technical replicates. As a consequence, all statistical analysis was based on one

1046 (transversal) imaging orientation. In order to provide a universal readout for each subject, a mean

1047 MSOT signal was calculated over all anatomical regions. This was used for correlations to

1048 clinical standard testing.

1049

1050 Data availability statement

1051 The data sets generated during and/or analyzed during the current study are available from the

1052 corresponding author on reasonable request. Restrictions may apply due to patient privacy and

1053 the General Data Protection Regulation.

1054

1055

\section{Methods-only references}

1057

1058

1059

1060

1061

1062

1063

1064

1065

1066

1067

1068

1069

1070

1071

1072

1073

1074

1075

1076

1077

61. Schindelin, J., et al. Fiji: an open-source platform for biological-image analysis. Nat Methods 9, 676-682 (2012).

62. Cox, J. \& Mann, M. MaxQuant enables high peptide identification rates, individualized p.p.b.-range mass accuracies and proteome-wide protein quantification. Nat. Biotechnol. 26, 1367-1372 (2008).

63. Tyanova, S., et al. The Perseus computational platform for comprehensive analysis of (prote)omics data. Nat. Methods 13, 731-740 (2016).

64. R Core Team. R: A Language and Environment for Statistical Computing. (R Foundation for Statistical Computing, Vienna, Austria, 2018).

65. Backman, M., et al. Multi-omics insights into functional alterations of the liver in insulin-deficient diabetes mellitus. Mol Metab 26, 30-44 (2019).

66. Brooke, M.H., et al. Clinical trial in Duchenne dystrophy. I. The design of the protocol. Muscle Nerve 4, 186-197 (1981).

67. Ozalevli, S., Ozden, A., Itil, O. \& Akkoclu, A. Comparison of the Sit-to-Stand Test with 6 min walk test in patients with chronic obstructive pulmonary disease. Respir Med 101, 286-293 (2007).

68. Fowler, W.M., Jr., et al. Profiles of neuromuscular diseases. Design of the protocol. Am J Phys Med Rehabil 74, S62-69 (1995).

69. Heckmatt, J.Z., Leeman, S. \& Dubowitz, V. Ultrasound imaging in the diagnosis of muscle disease. $J$ Pediatr 101, 656-660 (1982).

70. $\quad$ Pillen, S., Arts, I.M. \& Zwarts, M.J. Muscle ultrasound in neuromuscular disorders. Muscle Nerve 37, 679693 (2008). 


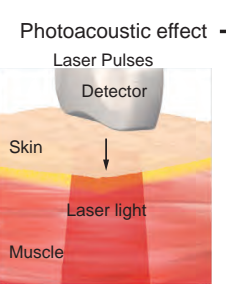

Ultrasound Waves

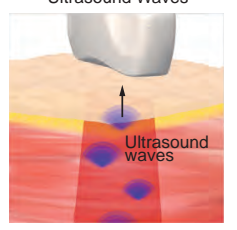

C

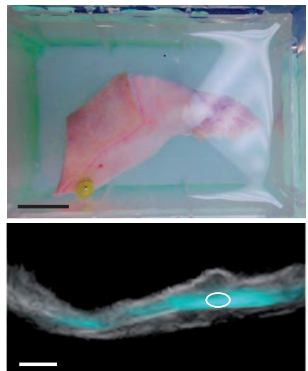

RUCT
Collagen

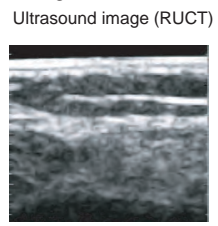

Single wavelength MSOT images

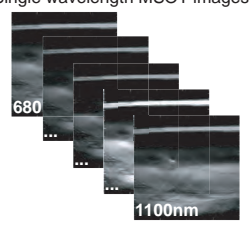

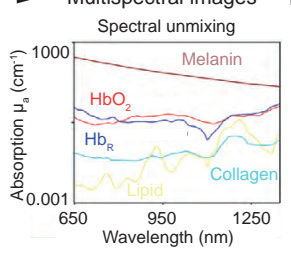
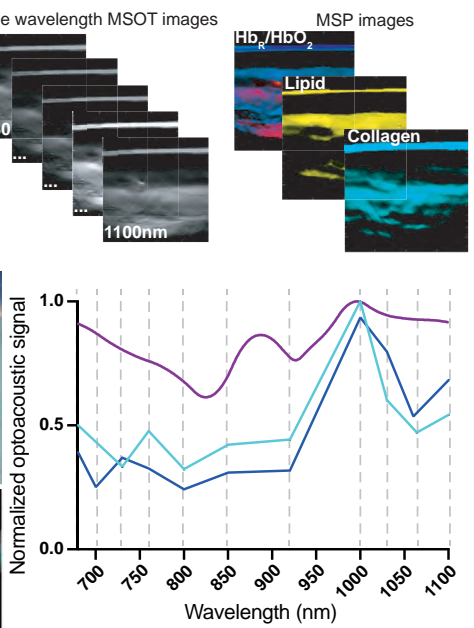

Collagen literature - Phantom collagen I
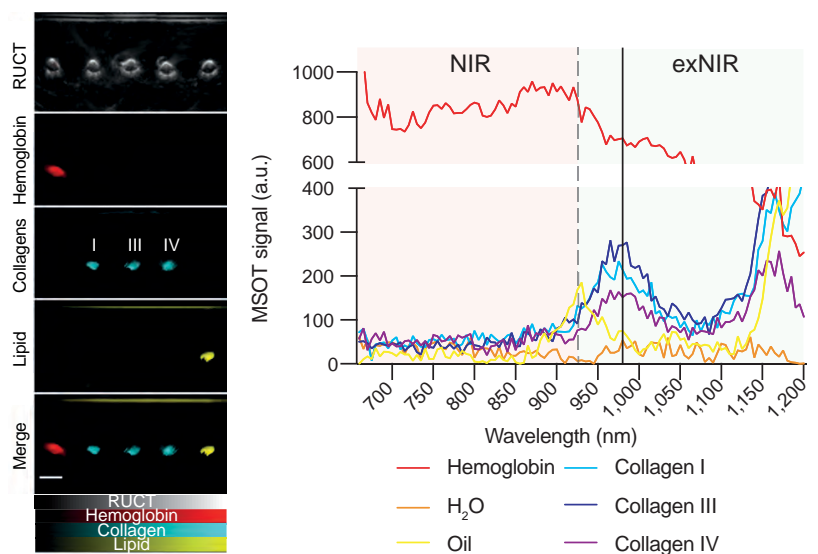

- Hemoglobin — Collagen I

$-\mathrm{H}_{2} \mathrm{O} \quad-$ Collagen III

Oil $\quad$ Collagen IV

d

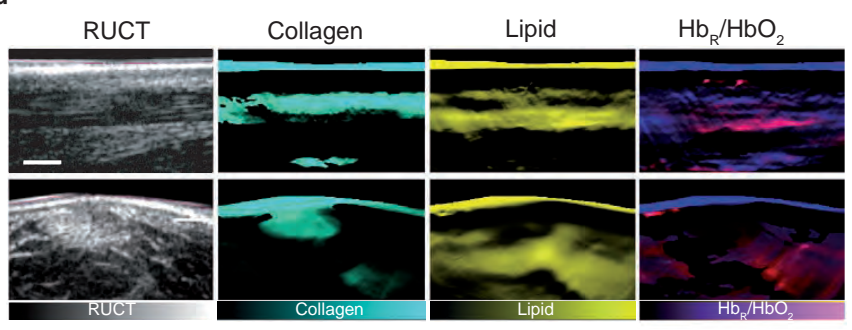
— ex vivo tendon 
$5 \sqrt{6}$

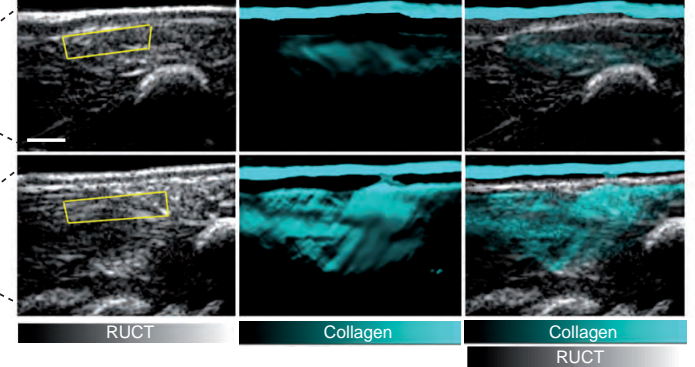

mean $2 \mathrm{D}$ collagen mean $2 \mathrm{D}$ collagen mean $2 \mathrm{D} \mathrm{Hb}$

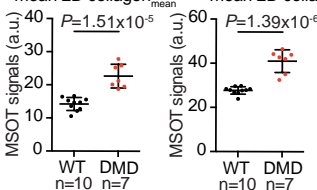

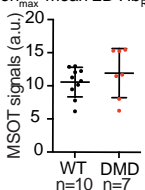

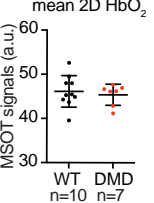

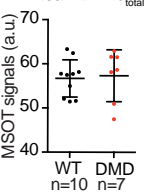

c

$\mathrm{ROC}$ of $2 \mathrm{D}$ Collagen ${ }_{\text {mean }}$

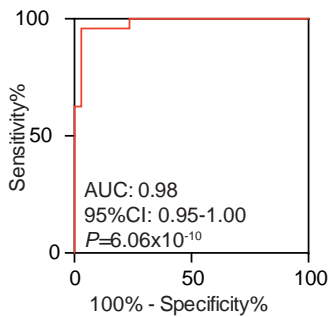

Trichrome staining

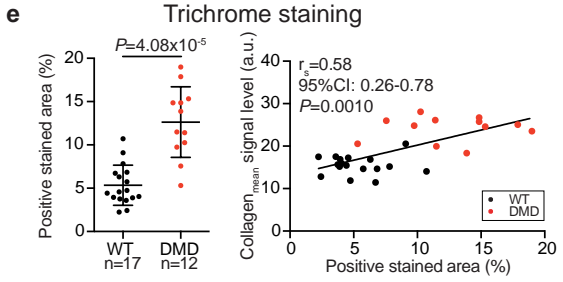

d $\quad H \& E(10 x / 40 x)$

ROC of $2 \mathrm{D}$ Collagen max $_{\text {max }}$

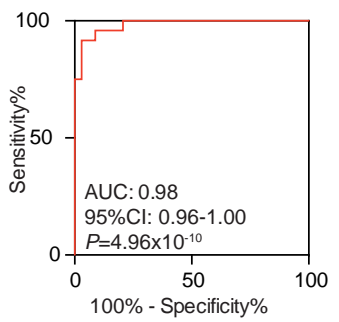

$\operatorname{TriC}(10 \mathrm{x} / 40 \mathrm{x})$

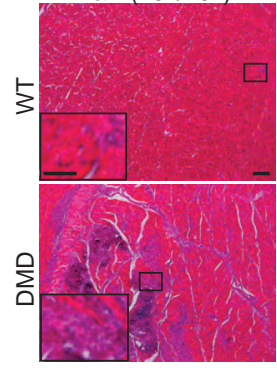

Sirius Red staining

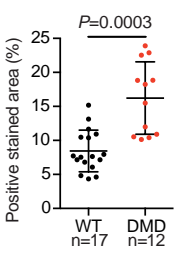

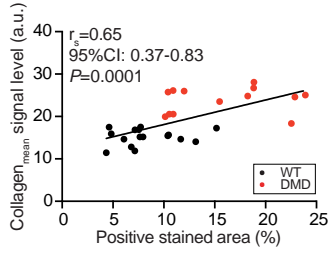

g
Dys1 (40x/80x)

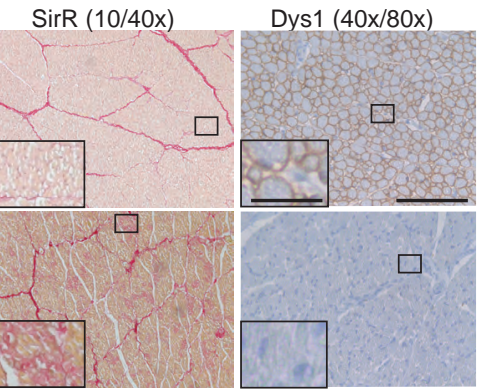

Total collagen/total protein content
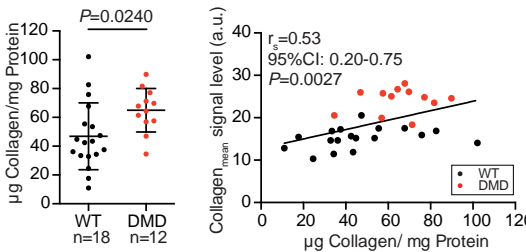


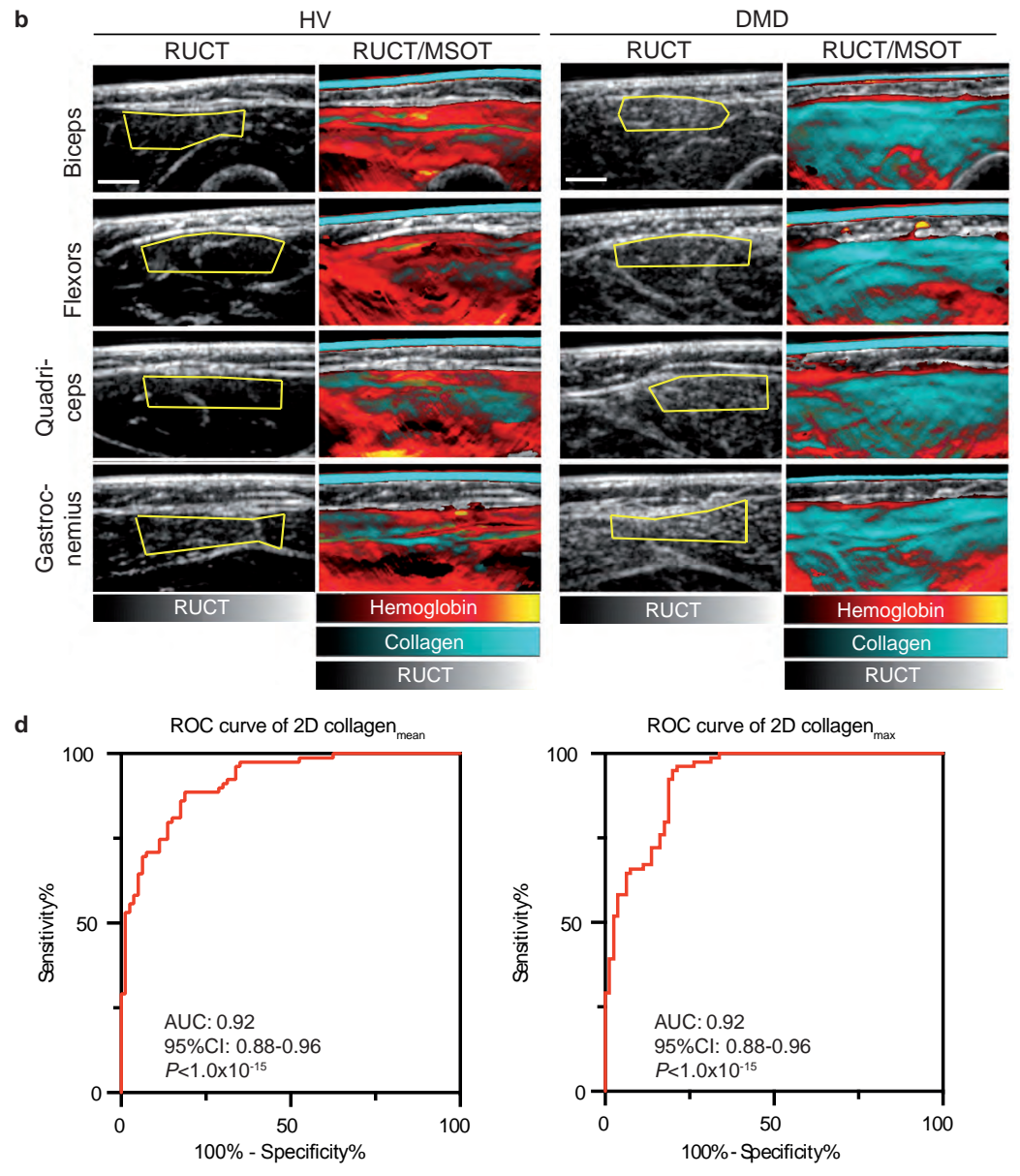




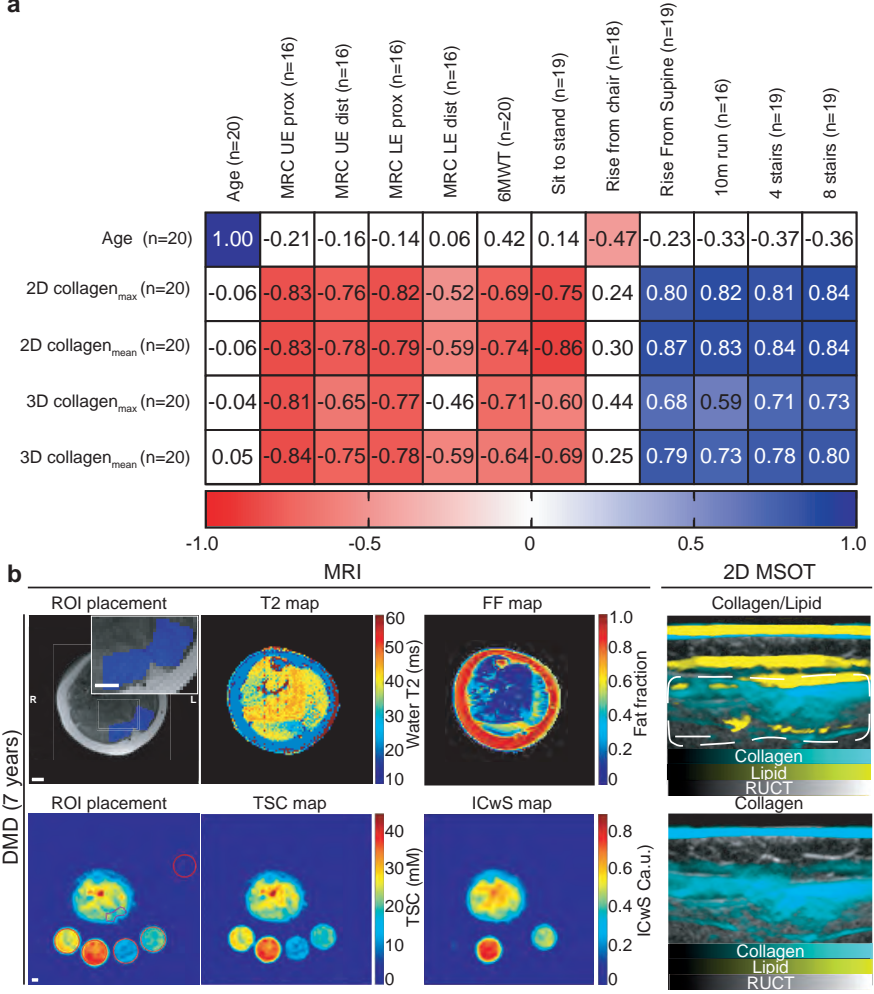


$\mathbf{a}$

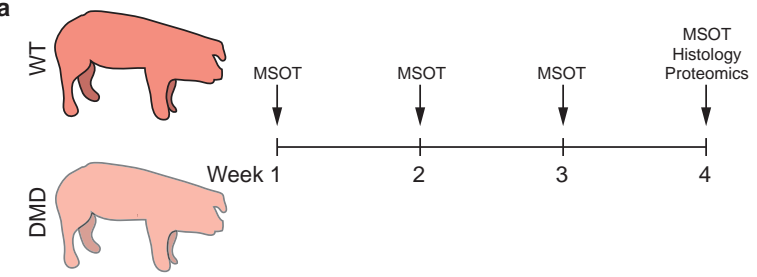

b

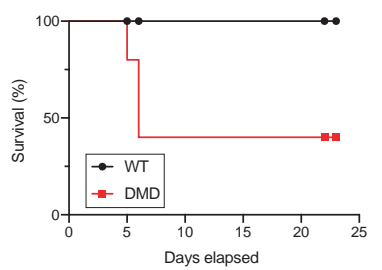

e 2D collagen mean All scans

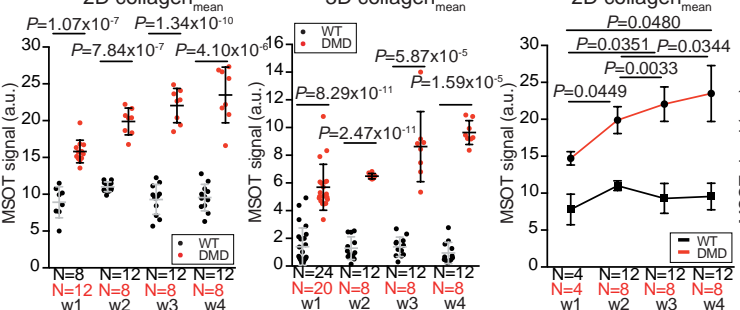

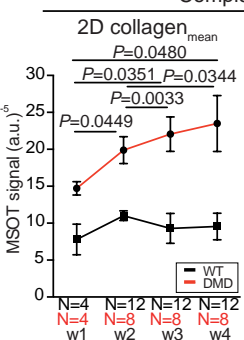

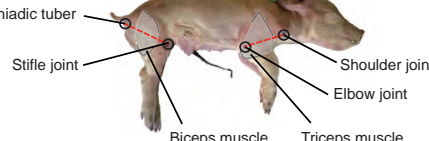

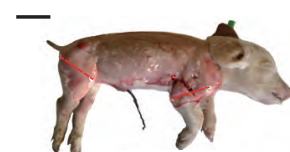

Completed scans

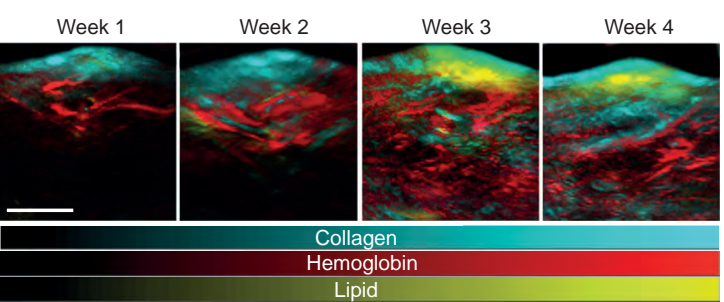

g Macroscopy

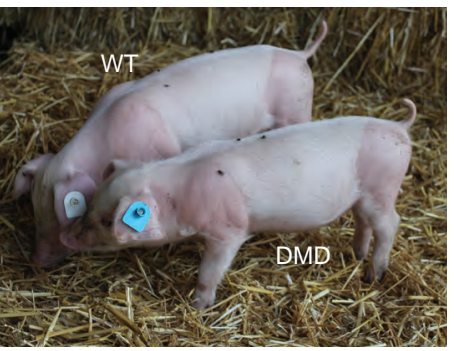

i

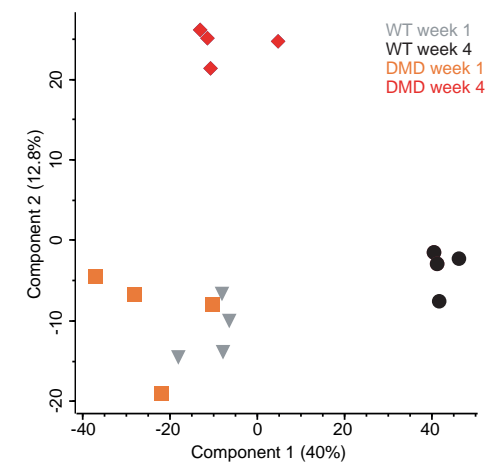

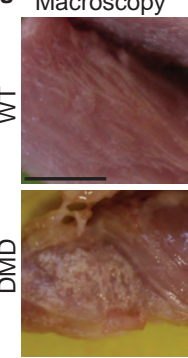

j

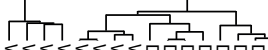

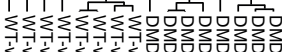

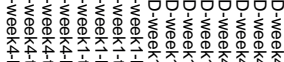

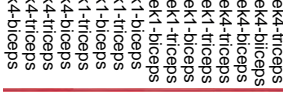

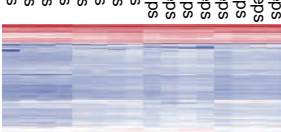

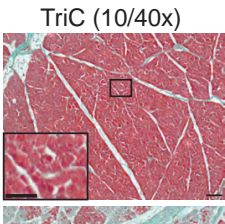

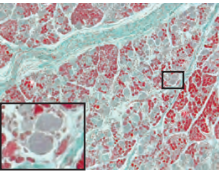

k

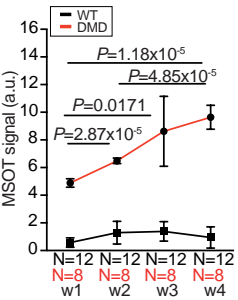

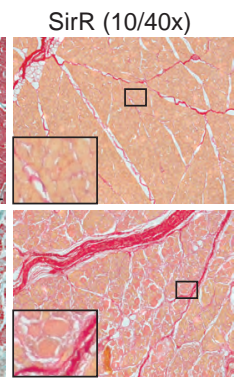

h Trichrome staining Trichrome staining

Sirius Red staining $P=3.63 \times 10^{-5}$
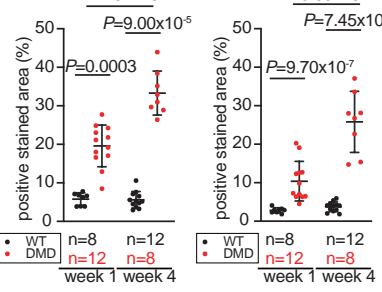

Total collagen total protein content

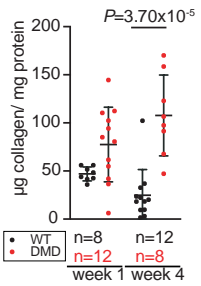

Week 1

Week 4
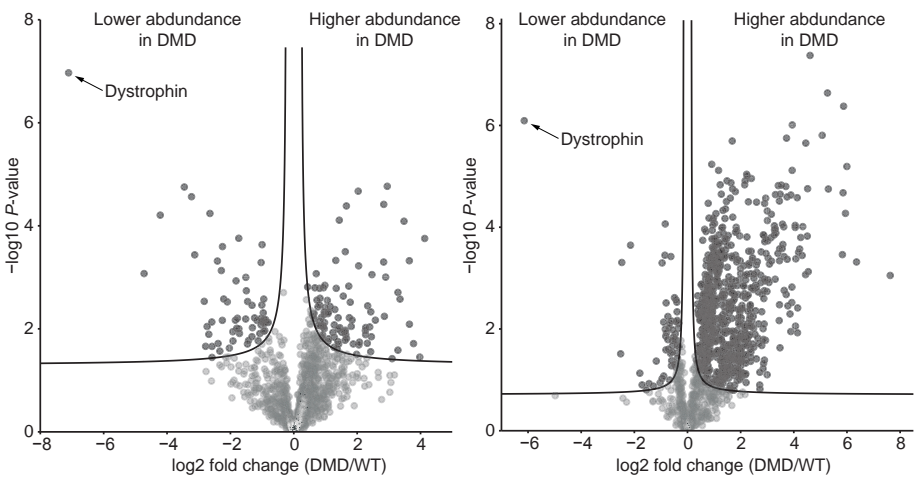
a

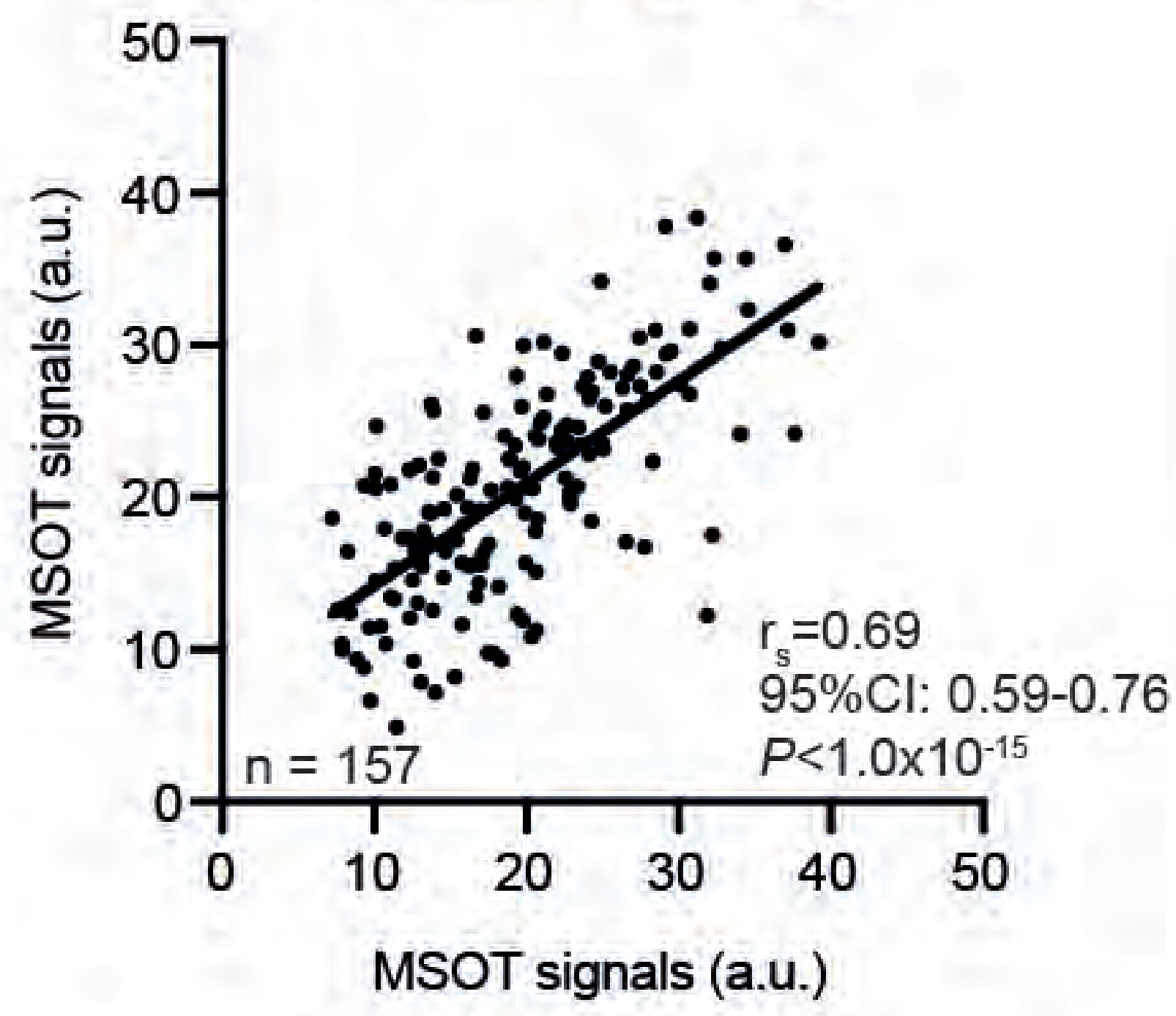

b 2D collagen $n_{\max }$

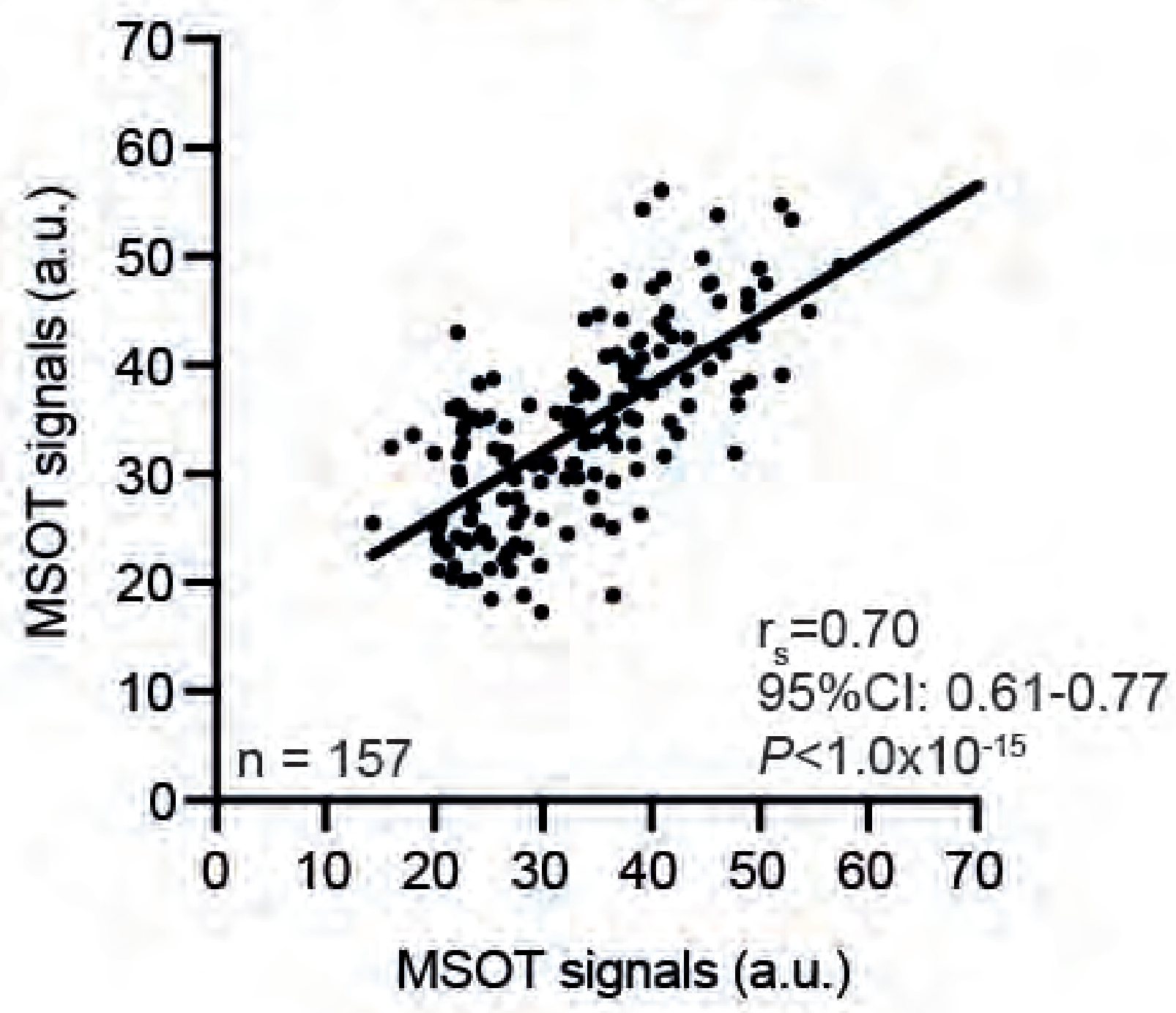


transversal

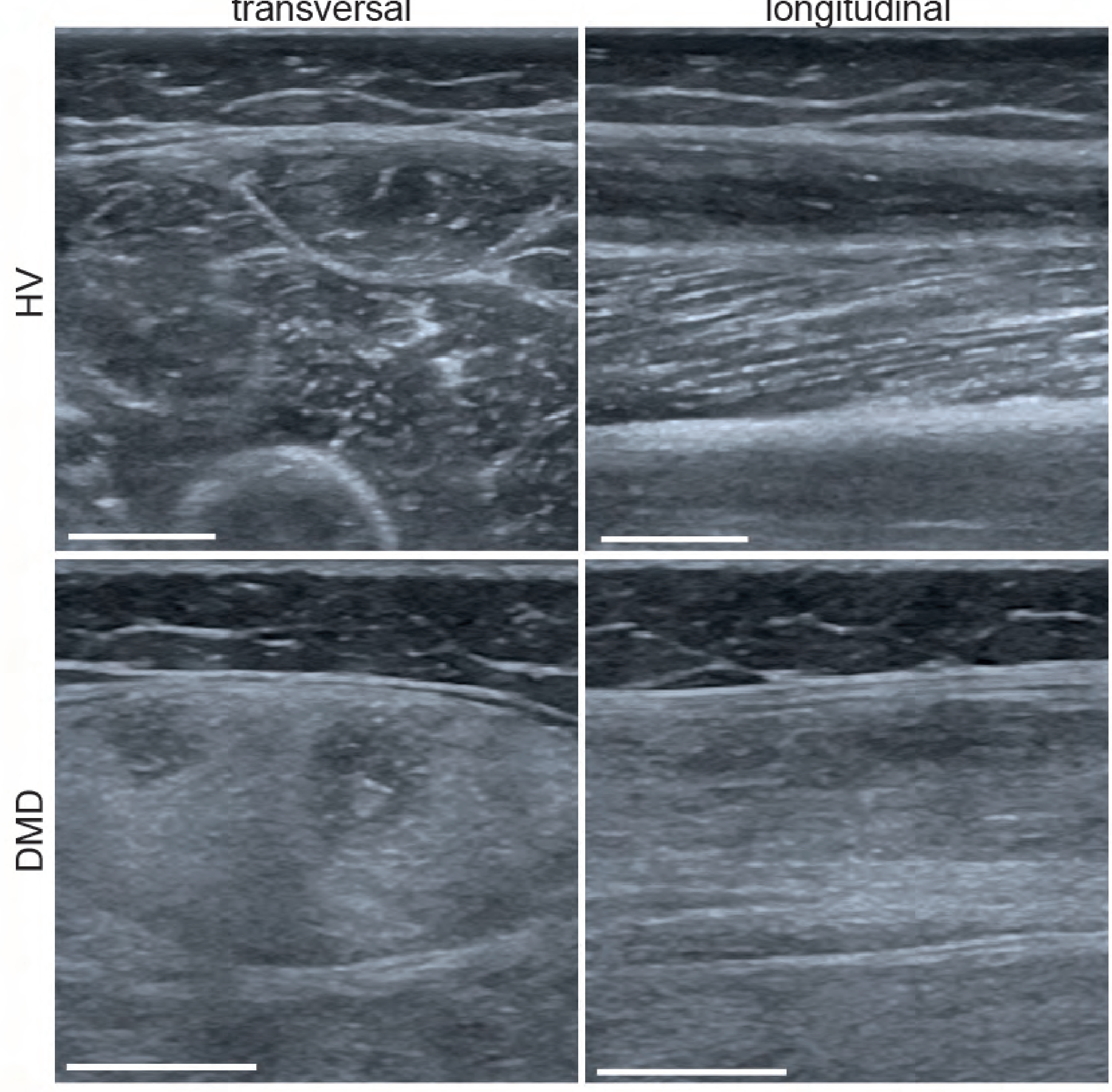


a 2D collagen $n_{\max }$

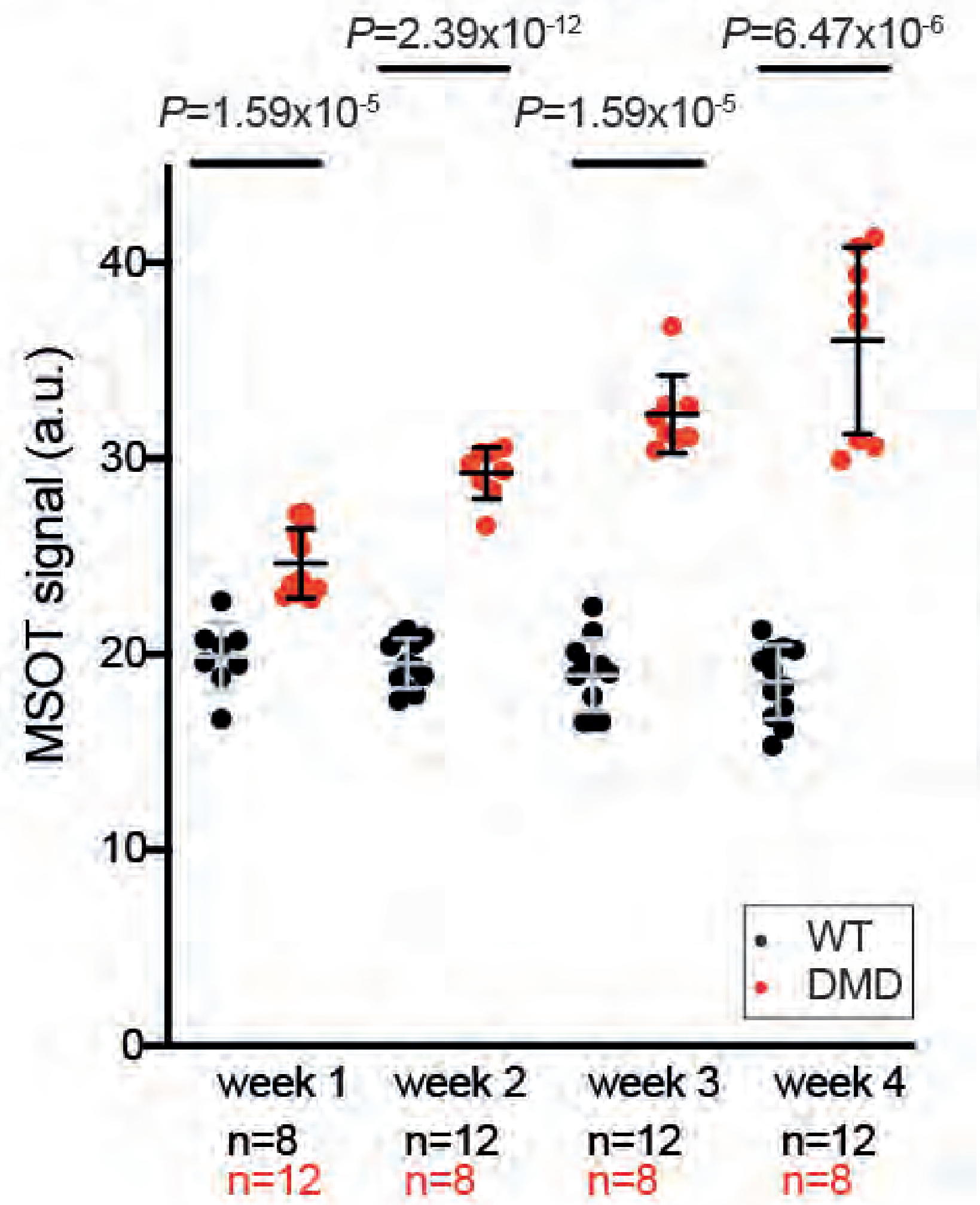

b

3D collagen ${ }_{\max }$

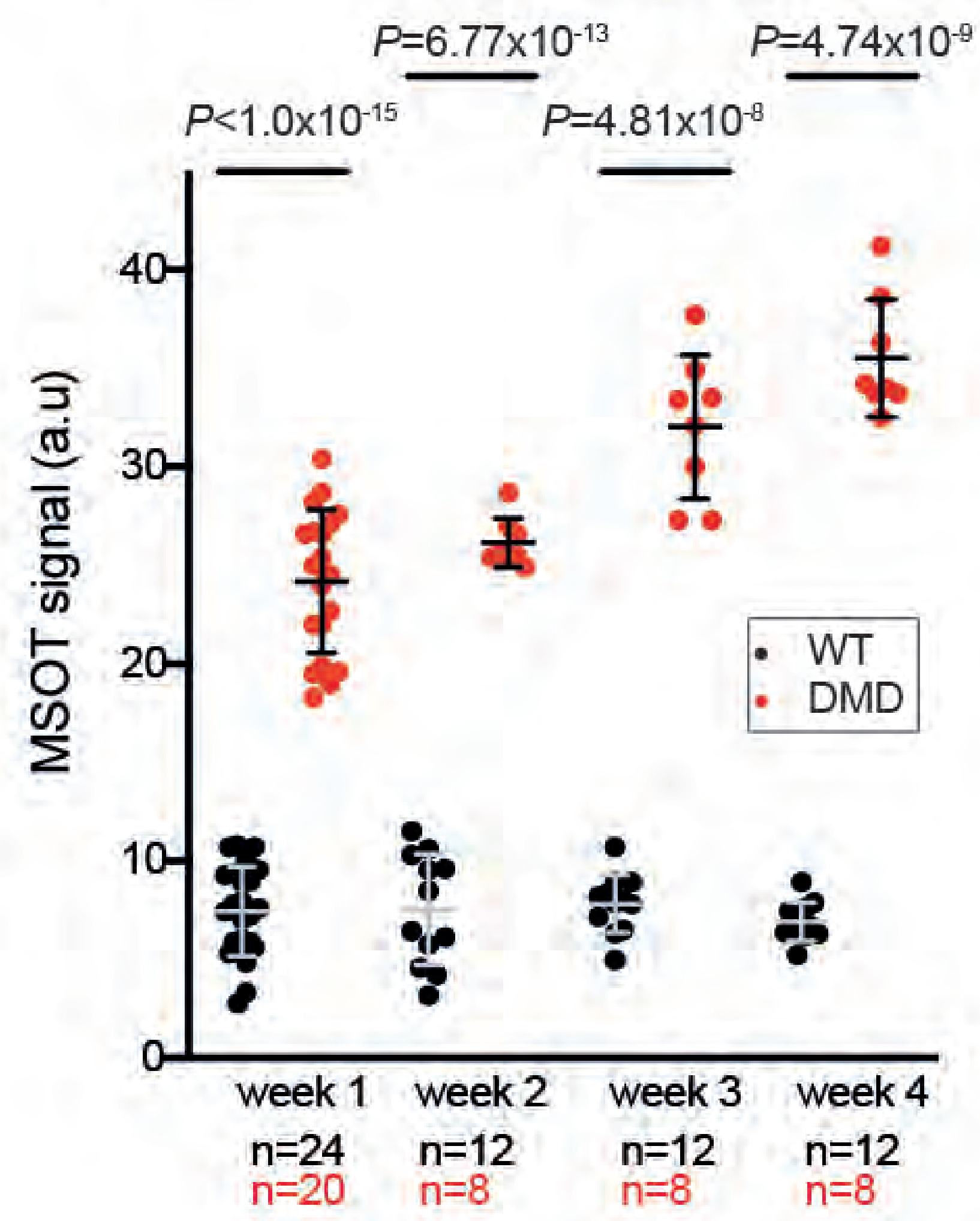


a

2D collagen $n_{\max }$

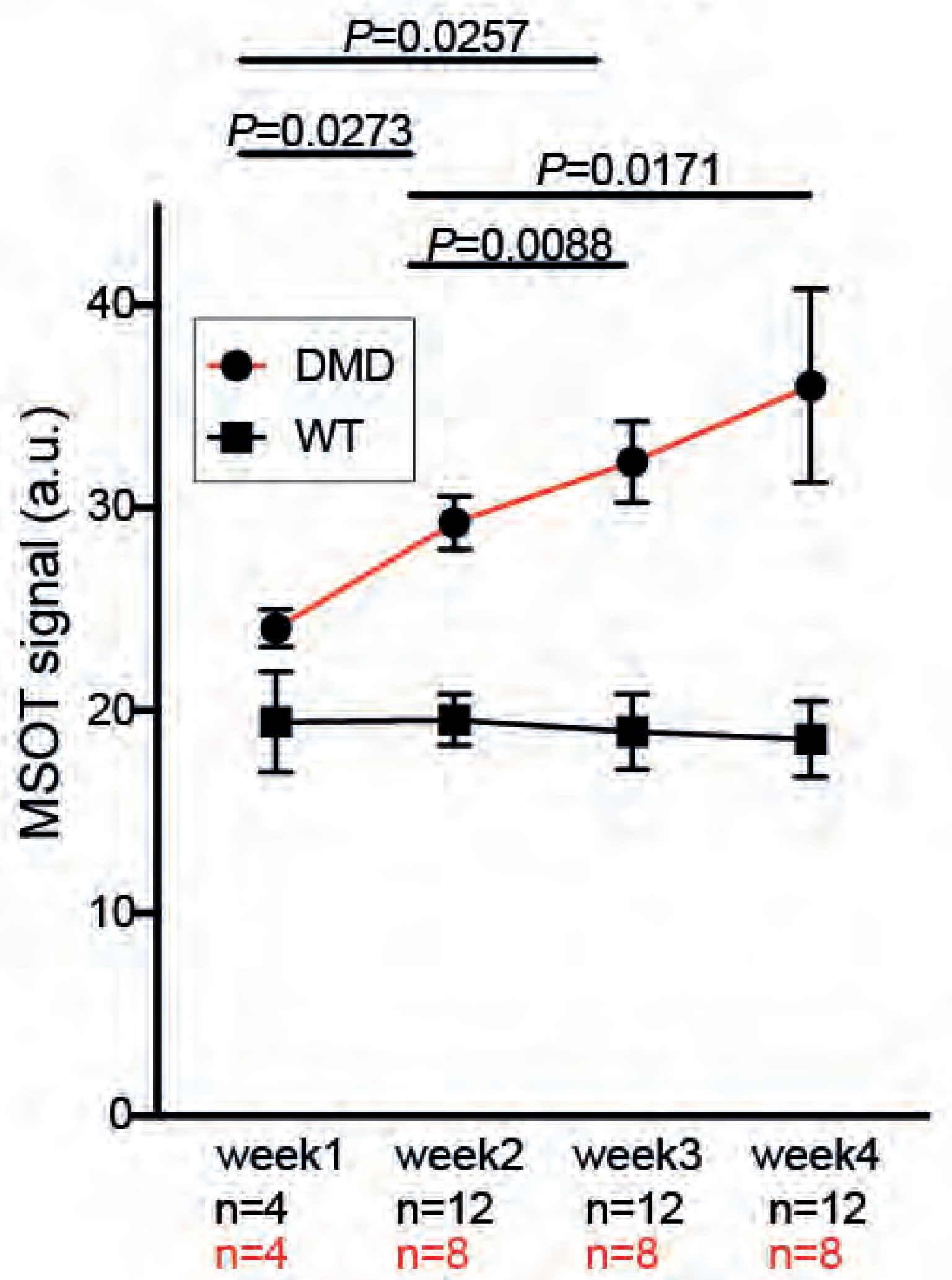

b

3D collagen ${ }_{\text {max }}$

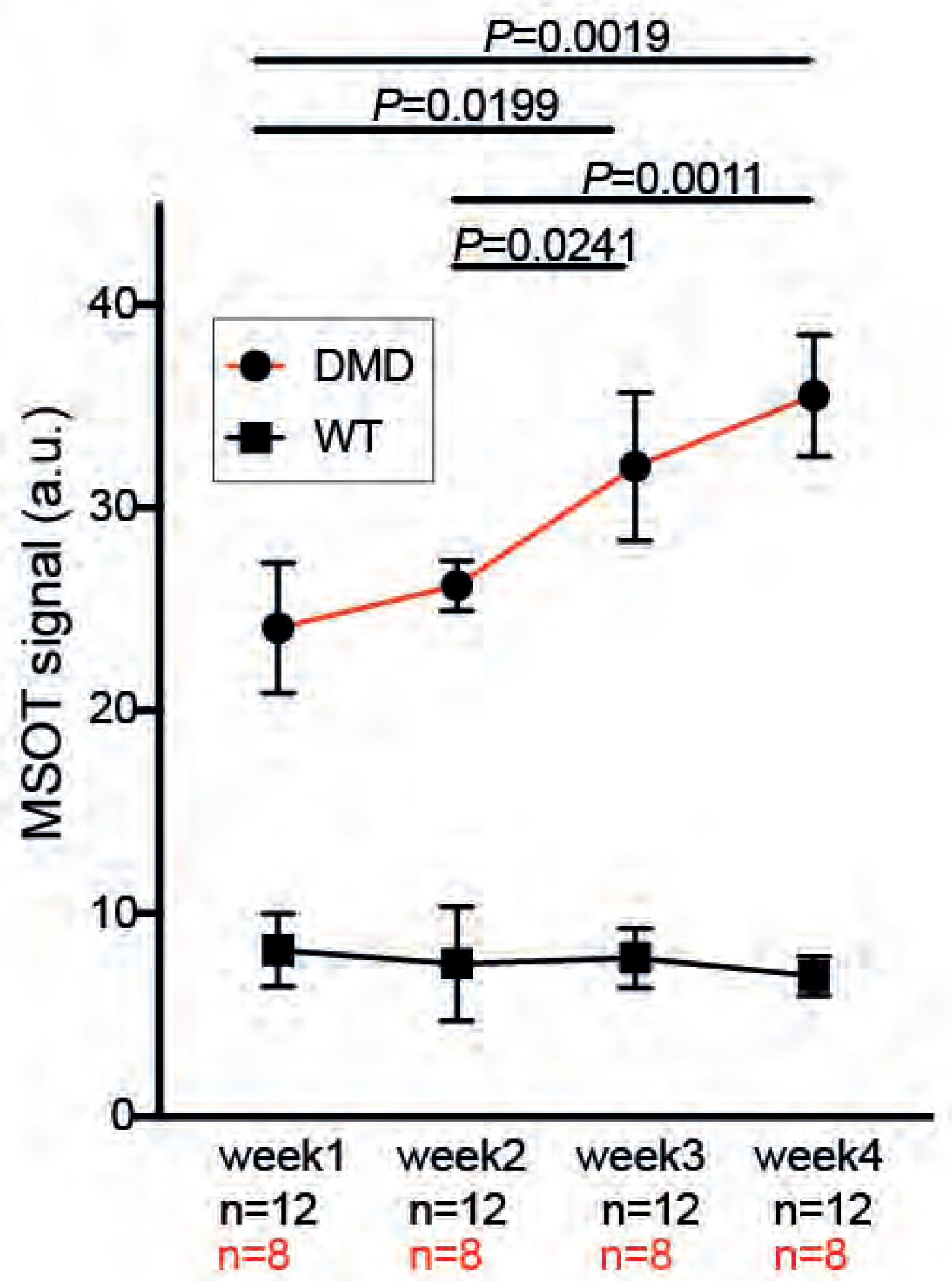




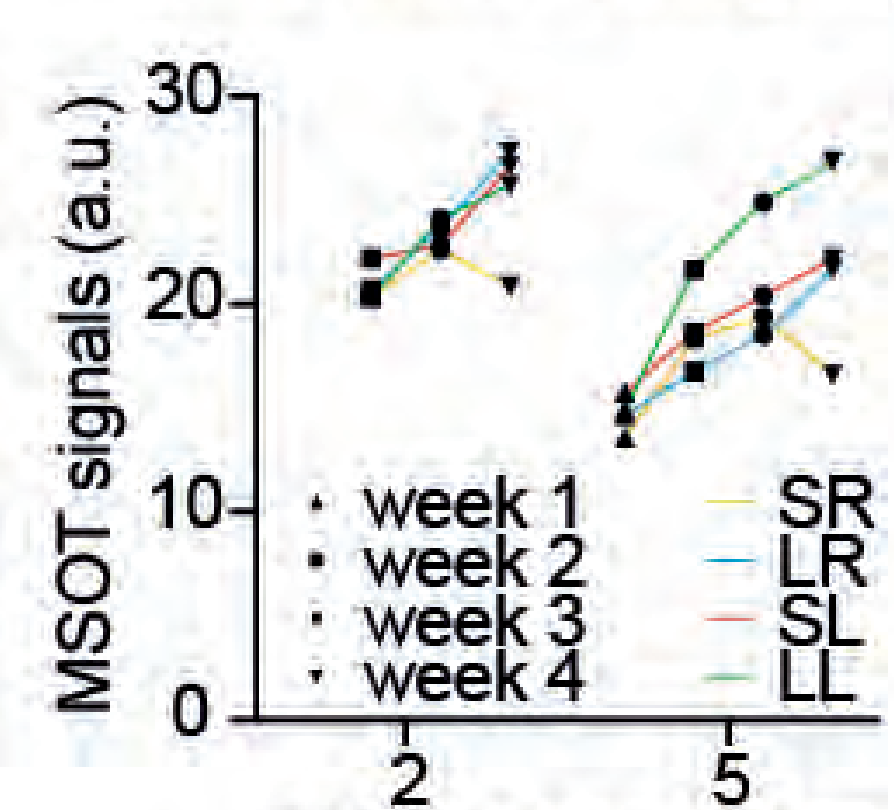

DMD piglet number $n=4$

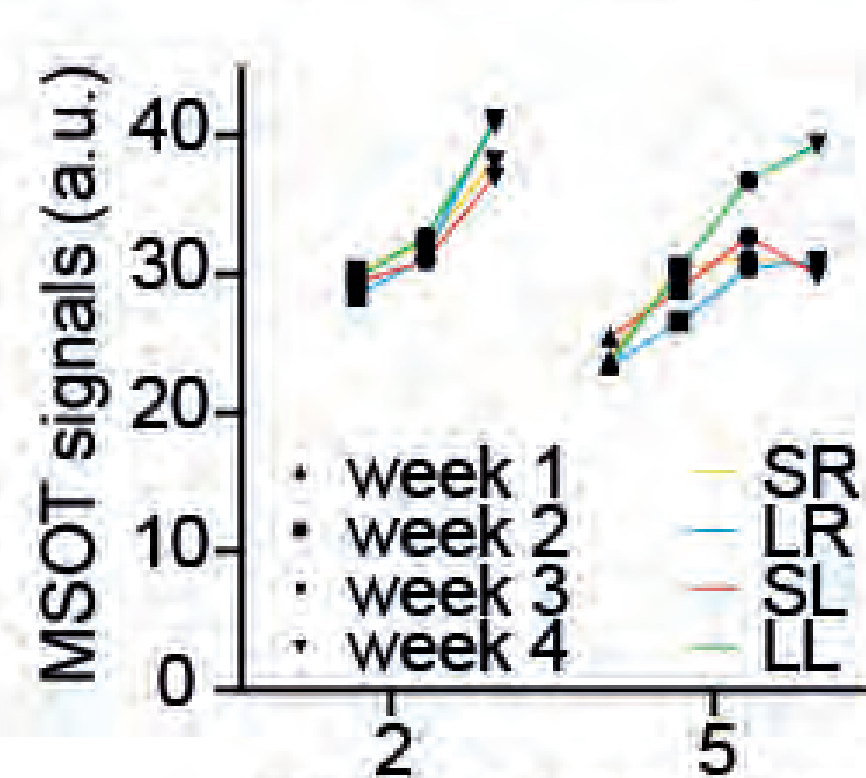

DMD piglet number $n=4$

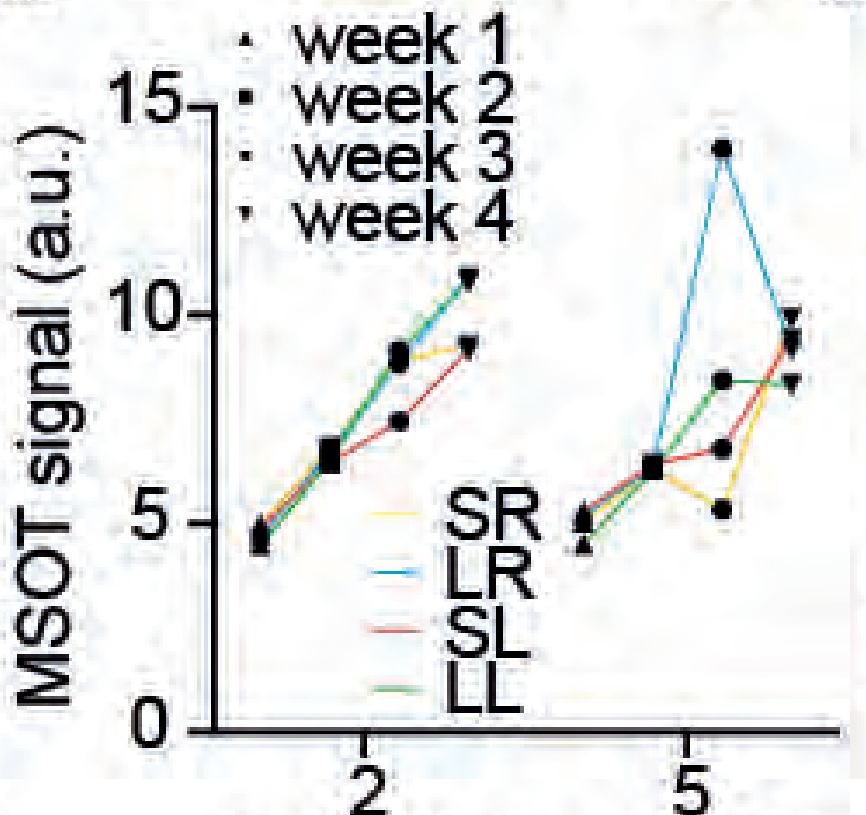

DMD piglet number $n=4$

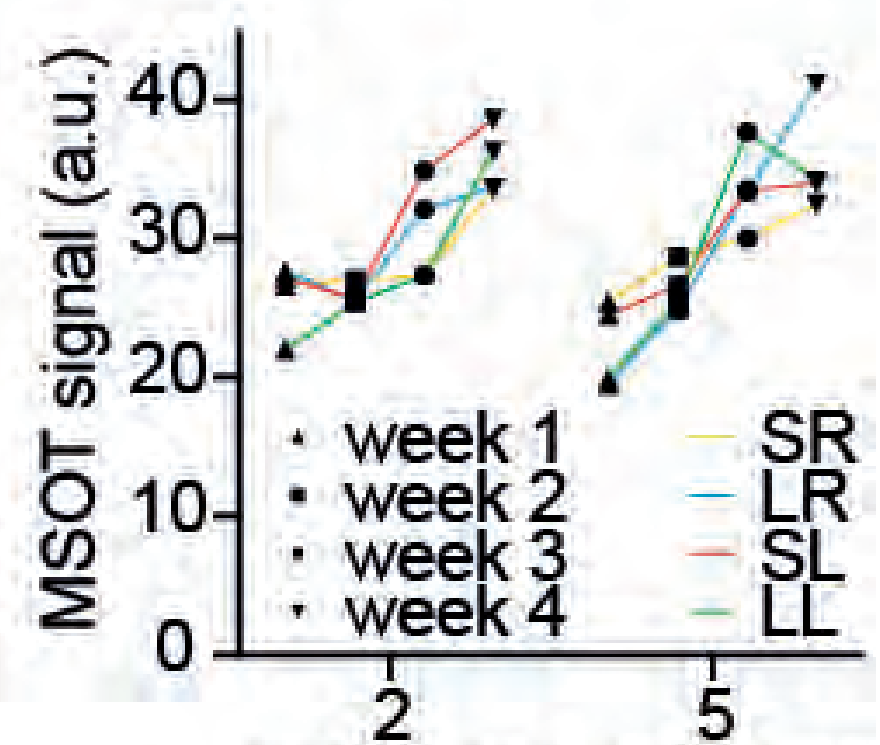

DMD piglet number $n=4$ $n=4$ 


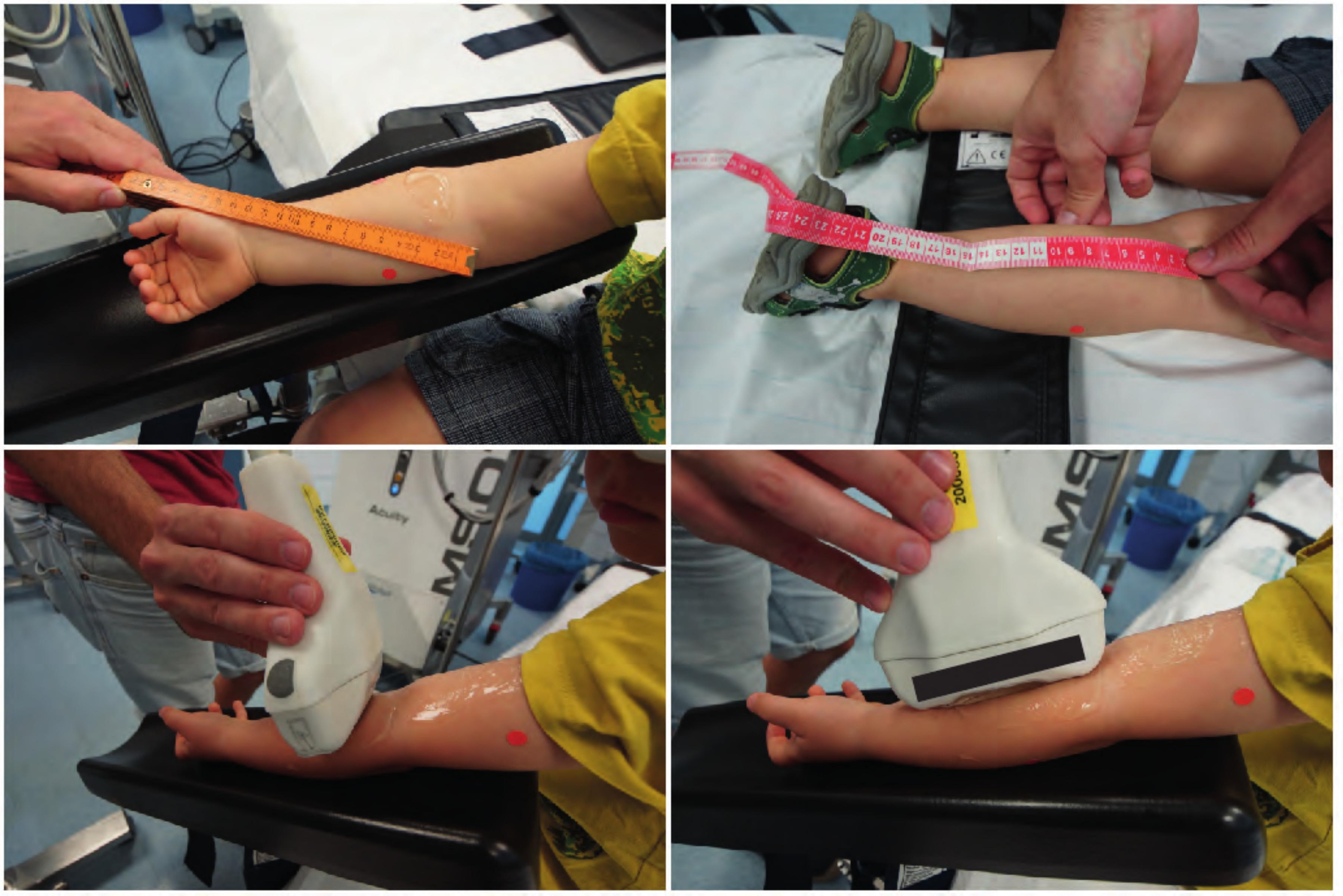

